\title{
NGAL decreases E-cadherin-mediated cell-cell adhesion and increases cell motility and invasion through Rac1 in colon carcinoma cells
}

\author{
Limei Hu${ }^{1}$, Walter Hittelman², Tao Lư ${ }^{2}$, Ping Ji ${ }^{1}$, Ralph Arlinghaus ${ }^{3}$, Ilya Shmulevich ${ }^{4}$, Stanley R Hamilton ${ }^{1}$ \\ and Wei Zhang ${ }^{1}$
}

Expression of neutrophil gelatinase-associated lipocalin (NGAL)/lipocalin2, a recently recognized iron regulatory protein that binds to matrix metalloproteinase-9 (MMP9), is increased in a spectrum of cancers, including those of the colorectum. Using colon carcinoma cell lines stably transfected with NGAL or antisense NGAL, we showed that NGAL overexpression altered subcellular localization of E-cadherin and catenins, decreased E-cadherin-mediated cell-cell adhesion, enhanced cell-matrix attachment, and increased cell motility and in vitro invasion. Conversely, a decrease in NGAL enhanced more aggregated growth pattern and decreased in vitro invasion. We further showed that NGAL exerted these effects through the alteration of the subcellular localization of Rac1 in an extracellular matrix-dependent, but MMP9-independent, manner. Furthermore, we observed that the NGAL-overexpressing cells tolerated increased iron levels in the culture environment, whereas the NGAL-underexpressing cells showed significant cell death after prolonged incubation in highiron condition. Thus, overexpressing NGAL in colon carcinomas is an important regulatory molecule that integrates extracellular environment cues, iron metabolism, and intracellular small GTPase signaling in cancer migration and invasion. NGAL may therefore be a new target for therapeutic intervention in colorectal carcinoma.

Laboratory Investigation (2009) 89, 531-548; doi:10.1038/labinvest.2009.17; published online 23 March 2009

KEYWORDS: NGAL; colon cancer; invasion; Rac1; E-cadherin; MMP9

Neutrophil gelatinase-associated lipocalin (NGAL)/lipocalin2 belongs to the lipocalin family, which includes over 20 proteins. ${ }^{1}$ It is a $25-\mathrm{kDa}$ protein that was originally discovered in human neutrophils and is known to form complexes through disulfide bonds with matrix metalloproteinase-9 (MMP9)., ${ }^{2,3}$ The best-known function of these lipocalin proteins is to serve as carriers for a myriad of small hydrophobic ligands. ${ }^{1,4}$

NGAL mRNA and protein have been found to be overexpressed in a broad spectrum of cancers, including breast, ovarian, pancreatic, colorectal, lung, urinary bladder, and hepatic tumors. ${ }^{5-10}$ Moreover, NGAL levels are elevated in the urine of breast and bladder cancer patients. ${ }^{5,8}$ Thus, it is hypothesized that NGAL may play a role in cancer pathophysiology.

It was speculated that NGAL stabilizes MMP9 and increases cell invasion, ${ }^{2,3}$ which is a common feature of cancer invasion as well as inflammation and tissue repair after injury. However, the function of NGAL is likely to be cell-typedependent. It was recently suggested that the Bcr-abl oncogene in chronic myeloid leukemia cells activates NGAL expression, which could cause apoptosis of normal hematopoietic cells. ${ }^{11,12}$ However, the mechanisms by which NGAL may contribute to solid tumor pathophysiology are obscure.

In this study, we showed by immunohistochemistry that NGAL is overexpressed in colorectal carcinoma cells. We next examined the potential underlying the molecular mechanism for the involvement NGAL in colorectal cancer. As a first step, we generated a set of colon carcinoma subclones from the KM12C cell line that either overexpressed or underexpressed NGAL and then used them to examine the relationship between NGAL expression and cell proliferation,

\footnotetext{
${ }^{1}$ Department of Pathology, The University of Texas MD Anderson Cancer Center, Houston, TX, USA; ${ }^{2}$ Department of Experimental Therapeutics, The University of Texas MD Anderson Cancer Center, Houston, TX, USA; ${ }^{3}$ Department of Molecular Pathology, The University of Texas MD Anderson Cancer Center, Houston, TX, USA and ${ }^{4}$ Institute for Systems Biology, Seattle, WA, USA

Correspondence: Professor W Zhang, PhD, or Dr SR Hamilton, MD, Department of Pathology, The University of Texas, MD Anderson Cancer Center, 1515 Holcombe Blvd., Houston, TX 77030, USA.
}

E-mail:wzhang@mdanderson.org or shamilto@mdanderson.org

Received 11 September 2008; revised 6 January 2009; accepted 2 February 2009 
death, adhesion, motility, and invasion. We observed that NGAL overexpression did not affect cell growth rate or death. However, it altered cellular cytoskeleton arrangement, cellcell and cell-matrix interactions, subcellular localizations of E-cadherin and catenin proteins, and tumor cell in vitro invasion. We showed that NGAL achieved these effects through the alteration of Rac1, one of Rho small GTPases. Further, we showed that extracellular matrix signal is required for NGAL to function, and iron also affects the function of NGAL. Knocking down NGAL in two other colon cancer cell lines also showed decreasing in vitro invasion.

\section{MATERIALS AND METHODS Antibodies, Plasmids, and Reagents}

Mouse monoclonal anti-NGAL (HYB 211-01) antibody was purchased from AntibodyShop (Fentofte, Denmark). The following antibodies were purchased from Santa Cruz Biotechnology Inc. (Santa Cruz, CA, USA): mouse monoclonal anti-RhoA, goat polyclonal anti-actin, rabbit polyclonal antiE-cadherin, mouse monoclonal anti-MMP9, and rabbit polyclonal anti-ferritin heavy chain. The following antibodies were purchased from BD Biosciences Pharmingen (Los Angeles, CA, USA): mouse monoclonal anti- $\alpha$-catenin, anti$\beta$-catenin, anti-transferrin receptor, anti-Rac1, anti-cdc42, anti-E-cadherin-FITC, anti-Rac1-FITC, and anti- $\beta$-cateninTRITC. Mouse monoclonal anti- $\alpha$-tubulin, deferoxamine (DFO), and ferric chloride were purchased from Sigma-Aldrich (Saint Louis, MO, USA). The dominant-negative Rac1GFP (DN Rac1-GFP) and constitutively active Rac1-GFP (CA Rac1-GFP) plasmids were generous gifts from Dr G Bokoch (Scripps Research Institute, La Jolla, CA, USA). The pcDNA3.1 $(+)$ plasmid was purchased from Invitrogen (Carlsbad, CA, USA). Transfection reagent and the Nucleofector device were purchased from Amaxa Inc. (Gaithersburg, MD, USA). The MMP9-lipocalin complex from human neutrophils was purchased from Calbiochem (catalog no. 444233; Calbiochem, San Diego, CA, USA).

\section{Generation of NGAL Expression Constructs}

The human NGAL full-length cDNA was removed by EcoRI and XhoI restriction enzyme digestion from a commercial cDNA clone (catalog no. MHS1011-9496856; Open Biosystems) and subsequently cloned into the expression vector pcDNA3.1 $(+)$ (Invitrogen). The construct of the NGAL/ pDsRed-N2 with the antisense sequence was generated by insertion of a PCR product using the full-length cDNA as template into the pDsRed-N2 vector (BD). The sequences of the PCR primers were as follows: forward primer: $5^{\prime}$-CCGGAATTCCGGAGCCACCATGCCCCTAGGTCTCCTGT GGCTG-3' ${ }^{\prime}$ and reverse primer: $5^{\prime}$-CCGGAATTCCGGAGC CGT-CGATACACTGGTCGATTGG-3'. All constructs were verified by sequencing. The NGAL/pcDNA3 $(+)$ containing sense sequence was used for the generation of NGAL-overexpressing clones, and the NGAL/pDsRed-N2 containing antisense was used for the generation of NGAL-underexpressing clones.

\section{Cell Culture and Transfection}

The human KM12C colon carcinoma cell line was provided by Dr Isaiah Fidler (The University of Texas MD Anderson Cancer Center), and DLD1 and HCT116 cells were purchased from ATCC. Both KM12C and DLD1 cells were maintained in Dulbecco's modified Eagle's medium/high-glucose medium. HCT116 cells were maintained in McCoy's 5A medium. All cell culture media were supplemented with $10 \%$ fetal bovine serum (FBS) and antibiotics and all cells were cultured in a humidified incubator containing $5 \% \mathrm{CO}_{2}$ at $37^{\circ} \mathrm{C}$. $\mathrm{KM} 12 \mathrm{C}$ cells were transfected with the expression plasmids (sense and antisense NGAL) and, as a control, with the empty pcDNA3.1 $(+)$ vector. Both transient and stable transfections were carried out with the Nucleofector device using the solution $\mathrm{T}$ and $\mathrm{T}_{20}$ program (Amaxa Biosystems, Cologne, Germany) according to the manufacturer's instructions. To establish cell lines stably expressing NGAL, the transfected cells were subsequently selected in Geneticin (G418)-containing medium $(600 \mu \mathrm{g} / \mathrm{ml})$ for 4 weeks after $48 \mathrm{~h}$ of transfection. After selection, a mixture of G418-resistant vectortransfected cells was collected and expanded. Several G418resistant clones from NGAL-transfected cells were isolated and transferred to a 24-well plate for expansion. The isolated colonies were screened for NGAL expression through western blotting of cell extracts with anti-NGAL antibody. Two NGAL-overexpressing clones (N11 and N15) generated by the NGAL/pcDNA3.1 $(+)$ expression construct and two NGAL-underexpressing clones (N19 and N22) generated by the NGAL/pDsRed-N2 (antisense) construct were chosen. As a similar cell morphology was observed for the cells transfected with the empty vectors of pcDNA3.1 $(+)$ and pDsRed$\mathrm{N} 2$, the pcDNA3.1 $(+)$ cells were used as vector control.

\section{SiRNA Transfection}

Double-strand NGAL siRNA was purchased from Ambion, with the sequence (sense) as follows: 5'-AAGTGGTA TGTGGTAGGCCTG- $3^{\prime}$. The negative non-targeting control siRNA was purchased from Qiagen (catalog no. 1027311). The siRNA transfection for DLD1 and HCT116 cells was performed according to the manufacturer's instruction using Lipofectamine 2000 Reagent (Invitrogen). Briefly, $3 \times 10^{5}$ cells in $2 \mathrm{ml}$ of complete medium were seeded into each well of an un-coated six-well plate $24 \mathrm{~h}$ before transfection. After $24 \mathrm{~h}$ of incubation, the medium was changed to $800 \mu \mathrm{l}$ of serum-free medium, and $50 \mu \mathrm{M}$ of NGAL siRNA or negative control siRNA in $200 \mu \mathrm{l}$ of serum-free DMEM containing $4 \mu \mathrm{l}$ of Lipofectamine 2000 Reagent was added into each correspondent well. After $4 \mathrm{~h}$ of incubation, $1 \mathrm{ml}$ of complete medium was added into each well and the cells were continually incubated. Duplicate experiments were performed. The cell lysates from one set of the experiments were collected for western blot analysis $72 \mathrm{~h}$ post-transfection. The 
cells from the other set of the experiments were trypsinized out of the plate $48 \mathrm{~h}$ post-transfection and used for in vitro invasion assay as described later.

\section{Cell Adhesion Assay}

Parental and transfected KM12C cells (trypsinized with trypsin-EDTA) were seeded into type-IV collagen-coated 12 -well plates (BD Biosciences) at a density of $2 \times 10^{5}$ cells/ well and cultured for $2 \mathrm{~h}$ in complete medium at $37^{\circ} \mathrm{C}$ to allow cell attachment. The culture medium was collected and the cells were washed two times with phosphate-buffered saline (PBS). The medium and the PBS from each washing were mixed together, and the non-adherent cells from each well were counted under a light microscope. The experiment was performed in triplicate and repeated twice for each sample.

\section{In Vitro Invasion Assays}

The in vitro invasion assays were performed using human type-IV collagen $\left(10 \mu \mathrm{g} / \mathrm{cm}^{2}\right)$-coated transwell inserts with an $8-\mu \mathrm{m}$ pore size for 24 -well plates (BD Biosciences). In this experiment, $750 \mu \mathrm{l}$ of medium with 10\% FBS was placed in the bottom wells of the plate. Next, $500 \mu \mathrm{l}$ of serum-free medium containing $3 \times 10^{4}$ cells were placed in triplicate inserts. The cells were allowed to invade through the matrix at $37^{\circ} \mathrm{C}$ in $5 \% \mathrm{CO}_{2}$ in a humidified incubator for $22 \mathrm{~h}$. The cells on the upper surface were removed by a cotton swab. Cells that had invaded to the lower surface of the filter were fixed and stained with HEMA-DIFF solution (Fisher, Pittsburgh, PA, USA). Cell invasion was quantified by counting the cells in the whole filter under a light microscope. All assays were performed in triplicate and repeated at least two times.

\section{Time-Lapse Live-Cell Imaging}

The NGAL-overexpressing and -underexpressing cells were cultured on human collagen-IV $\left(10 \mu \mathrm{g} / \mathrm{cm}^{2}\right)$-coated or -uncoated glass-slide chambers at a density of $2 \times 10^{4}$ per $\mathrm{cm}^{2}$ and incubated in complete medium for $24 \mathrm{~h}$. Then the medium was changed to serum-free, and live-cell images were captured with an Olympus inverted microscope (IX-81; Melville, NY, USA) using a $\times 10$ phase objective at 20 -min intervals for about $18 \mathrm{~h}$. The cells were incubated at $37^{\circ} \mathrm{C}$ in $5 \% \mathrm{CO}_{2}$. MetaMorph software (Molecular Devices, Downingtown, PA, USA) was used for microscope control, image acquisition, and image analysis.

\section{Western Blot Analysis}

Western blot assays were carried out as described earlier. ${ }^{13}$ Briefly, cell extracts containing $40-80 \mu \mathrm{g}$ of protein were resolved by $10 \%$ SDS-PAGE, transferred to Hybond ECL nitrocellulose membranes (Amersham Pharmacia Biotech, Chicago, IL, USA), blocked in 5\% skim milk in $1 \times$ Trisbuffered saline Tween-20, and probed with the primary antibodies at a concentration of 1:1000. The secondary antibodies were used at a concentration of 1:5000. The proteins were visualized using the Visualizer Western Blot Detection Kit (Upstate, Charlottesville, VA, USA). Protein fractionations were extracted using the NE-PER Nuclear and Cytoplasmic Extraction Reagents (Pierce, Rockford, IL, USA), and the manufacturer's instructions were followed.

\section{limmunofluorescence Staining}

The cells were fixed with $4 \%$ paraformaldehyde in PBS, permeabilized with PBS containing $0.1 \%$ Triton X-100 for $10 \mathrm{~min}$, and blocked with $10 \%$ goat serum for at least $1 \mathrm{~h}$ at room temperature. For single-antibody staining, the cells were incubated with fluorescence-conjugated antibody at a concentration of $1: 100$ for $1 \mathrm{~h}$ at room temperature. For double staining, the cells were incubated with the first antibody at a concentration of 1:100 for $1 \mathrm{~h}$ at room temperature or for $16-18 \mathrm{~h}$ at $4^{\circ} \mathrm{C}$. After washing, the cells were incubated with the fluorescence-conjugated secondary antibody at a concentration of $1: 100$ for $1 \mathrm{~h}$ at room temperature. After another washing, cells were counterstained with DAPI for fluorescence microscopy. Phalloidin staining was performed at room temperature for $45 \mathrm{~min}$ using Texas Red-conjugated phalloidin (Molecular Probes, Invitrogen) at a concentration of $0.5 \mu \mathrm{g} / \mathrm{ml}$. Images were captured by either confocal or phase-contrast fluorescence microscopy.

\section{Gelatin Zymographic Assay}

Gelatin zymographic assay was performed as described. ${ }^{13}$ Briefly, $5 \times 10^{5}$ cells were seeded onto a collagen-IV-coated six-well plate in DMEM/high glucose containing 10\% FBS and cultured for $24 \mathrm{~h}$. Cells were then washed twice with PBS and incubated with serum-free medium for $48 \mathrm{~h}$. The medium was collected and concentrated using YM-10 Amicon spin columns after the cell debris were removed by centrifugation. Equal amounts of the concentrated conditioned medium were mixed with SDS sample buffer without reducing agent and subjected to 7.5 SDS-PAGE containing $0.1 \%$ gelatin A. After electrophoresis, the gel was washed in $2.5 \%$ Triton X-100 four times ( $15 \mathrm{~min}$ each) at room temperature and then incubated for $24 \mathrm{~h}$ at $37^{\circ} \mathrm{C}$ in the buffer containing $5 \mathrm{mM} \mathrm{CaCl}_{2}$ and $1 \mu \mathrm{M} \mathrm{ZnCl}_{2}$. The proteins in the gel were stained with Coomassie Brilliant Blue.

\section{GTP-Rac1 Pull-Down Assays}

For all assays, $5 \times 10^{5}$ cells were cultured in collagen-IVcoated six-well plates (BD) in complete medium for $24 \mathrm{~h}$ and lysed after being washed twice with cold PBS in $25 \mathrm{mM}$ HEPES, pH 7.5; $150 \mathrm{mM} \mathrm{NaCl}$; 1\% Igepal CA-630; $10 \mathrm{mM}$ $\mathrm{MgCl}_{2} ; 1 \mathrm{mM}$ EDTA; $10 \%$ glycerol; and protease inhibitors. The Rac/cdc42 Assay Reagent (PAK-1 PBD, agarose conjugate) from Upstate was used to carry out the assay for GTPRac1 pull-down per the manufacturer's instructions. The GTP- and GDP-loaded samples were used as the positive and the negative controls, respectively. 


\section{Statistical Analysis}

Data are expressed as the mean \pm s.d. Statistical analysis was performed using Student's $t$-test. Differences in means were evaluated by a two-tailed $t$-test assuming unequal variances. A $P$-value $\leq 0.05$ was considered statistically significant.

\section{RESULTS}

NGAL is Overexpressed in Colorectal Carcinoma Tissues

In a pilot genomic profiling study, we compared gene expression profiles of 12 colorectal adenocarcinomas and 4 non-neoplastic colonic mucosal specimens and found NGAL among genes elevated in cancer tissues. To further confirm this result and examine whether NGAL is elevated at the protein level in malignant colorectal epithelial cells, we constructed a tissue microarray consisting of 16 non-neoplastic colorectal mucosa and 19 colorectal adenocarcinomas and evaluated the expression of NGAL using immunohistochemistry. We found that NGAL protein was not expressed in any of the normal colorectal mucosal specimens, but was present in the carcinoma cells of 16 of 19 tumors (Figure 1). In tissues in which both non-neoplastic and malignant epithelial cells were present (eg, panel $\mathrm{c}$ in Figure 1), we detected NGAL only in the carcinoma cells. The staining pattern of NGAL was heterogeneous and appeared intensely patchy in some cases and more evenly stained in other cases. NGAL expression was also detected in infiltrating macrophages in addition to the carcinoma cells. The surrounding matrix in the tumor tissues was also stained, which is consistent with the known secretion of NGAL. ${ }^{1}$ Thus, our findings are consistent with the earlier report in the literature. ${ }^{14}$

\section{NGAL Overexpression Alters Cytoskeletal Organization, Decreases Cell-Cell Adhesion, and Increases Cell-Matrix Adhesion}

To determine the consequences of NGAL upregulation in colon carcinoma cells, we transfected the NGAL expression construct with sense sequence into $\mathrm{KM} 12 \mathrm{C}$ cells to establish eight NGAL-overexpressing cell lines. As KM12C cells express a detectable level of endogenous NGAL, we also established four NGAL-underexpressing cell lines by transfection of the KM12C cells with the NGAL expression construct containing an antisense sequence. In addition, a mixture of empty vector-transfected $\mathrm{KM} 12 \mathrm{C}$ cell lines was also established to serve as control cells.
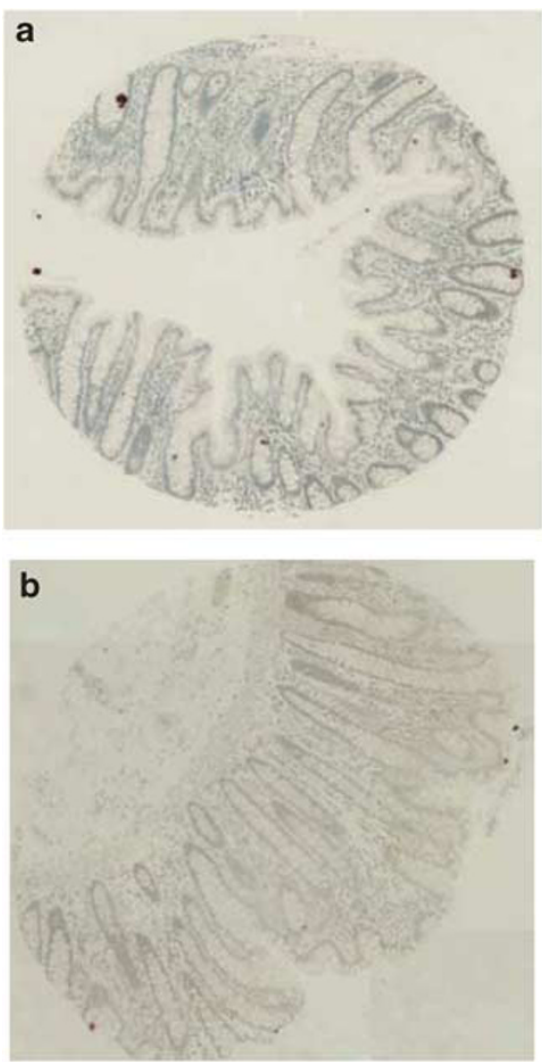
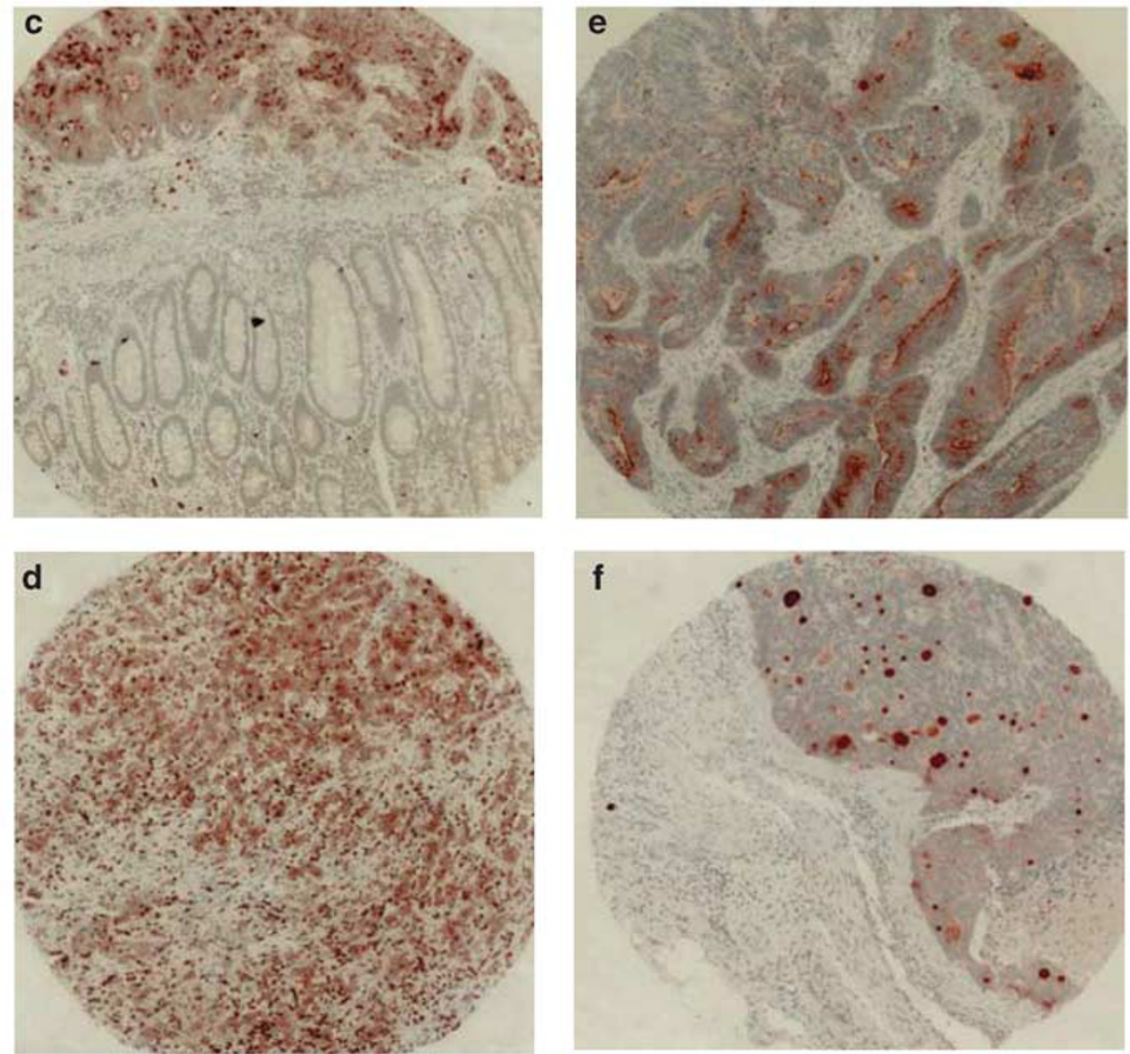

Figure 1 NGAL expression in colon carcinoma tissues. Tissue microarray consisting of 16 non-neoplastic colorectal mucosal specimens and 19 colorectal carcinoma tissues was constructed. The section was stained for the expression of NGAL using standard immunohistochemistry procedures. (a and b) Absence of staining for NGAL in the non-neoplastic mucosa. (c) A tissue section containing both carcinoma and non-neoplastic mucosa. NGAL is evident only in the carcinoma tissue. (d-f) Examples of staining for NGAL in the colorectal carcinomas. 
We randomly chose two of the NGAL-overexpressing clones (N11 and N15) and two NGAL-underexpressing clones (N19 and N22) (Figure 2a) to evaluate the effects of NGAL on the cells in our experiments. The morphology of the collagen-IV-adherent cells was first assessed by phasecontrast light microscopy. The vector-transfected control cells grew in tightly compact colonies that became several cell layers thick in the center (Figure 2b, left panel). As compared with the vector control cells, the NGAL-underexpressing cells showed an even more aggregated growth pattern with tight cell-cell adhesion (Figure 2b, center panel). These cells were also smaller in size compared with vector-transfected control cells (Figure 2b, center panel). However, the cells expressing NGAL at high levels showed loose cell-cell adhesion, formed more angular and flat colonies, and appeared more spread out at the edges where they were in contact with the substratum, exhibiting many spiky peripheral projections and lamellipodia (Figure 2b, right panel, arrowheads). The NGAL-overexpressing cells were bigger in size compared with the vector control cells (Figure $2 \mathrm{~b}$, right panel).

As these morphological changes suggest that NGAL affects cytoskeletal organization and cell-matrix contact, we examined the cytoskeleton structures by immunofluorescent staining for F-actin and tubulin, the two major cellular cytoskeletal components, using confocal and light microscopy, respectively. Phalloidin staining of the vector-transfected controls and the NGAL-underexpressing cells showed a cortical F-actin staining pattern in which the F-actin formed a thick actin cortex at cell-cell junctions and at the edges in contact with the substratum. In contrast, NGAL-overexpressing cells showed a more diffuse pattern of F-actin staining within cells and in cell membrane projections and lamellipodia (Figure 2c, upper right panel).

To examine the effects of NGAL overexpression on microtubule formation in the cells, we visualized the microtubular structures by immunofluorescence staining for $\alpha$-tubulin. The results were similar to those for the F-actin staining. In the vector-transfected and the NGAL-underexpressing cells, $\alpha$-tubulin also showed a peripheral staining pattern in which the protein was concentrated at cell-cell junctions and at the edges in contact with the substratum (Figure 2c, lower left and center panels). In addition, no significant microtubular structures were observed in these cells (Figure 2c, lower left and center panels). By contrast, $\alpha$-tubulin staining in the NGAL-overexpressing cells revealed prominent microtubular fibers extending from the microtubule-organizing center to the cell surface (Figure 2c, lower right panel). This, together with the flat and spread-out morphology assumed by the NGAL-overexpressing cells, suggests an increased adhesion of these cells to the matrix.

To examine the effects of NGAL upregulation on cellmatrix adhesion, we carried out a cell-attachment assay using plates coated with human type-IV collagen (described in Materials and methods). We chose this substrate because collagen IV is the major component of the colonic epithelial basement membrane. ${ }^{15,16}$ Results from the cell-attachment assay showed that $75-85 \%$ of NGAL-overexpressing cells (N11 and N15) attached to the plates after a 2-h incubation. By contrast, only $15-20 \%$ of parental cells, vector-transfected cells, and NGAL-underexpressing cells (N19 and N22) attached to the matrix during the same time period $(P<0.001$, Figure $2 \mathrm{~d}$ ). These results suggest that NGAL overexpression was associated with an increased cell-matrix adhesion.

\section{NGAL Overexpression Increases the Detachment of KM12C Cell From Colonies and In Vitro Invasion}

As dynamic regulation of the F-actin network is critical to cell motility, ${ }^{17}$ we hypothesized that the F-actin reorganization induced by NGAL overexpression might increase the motility of the tumor cells. We performed time-lapse live-cell imaging analysis to observe cell movement. Cells were first seeded on type-IV collagen-coated glass slides in complete culture medium for $24 \mathrm{~h}$ and then changed to serum-free medium. Images were taken at 20-min intervals up to $18 \mathrm{~h}$. The timelapse digital microscopy showed that the NGAL-underexpressing cells formed tight clusters and the cells rarely broke away from the cell colonies. Occasionally, cells broke free from a cell colony, but the free cells were rapidly 'captured' by another cell colony (Figure 3a, upper panel, and Supplementary movie M1). In contrast, the NGAL-overexpressing cells formed loose colonies, and many cells exhibited the freedom to move about without being constrained by a colony (Figure 3a, center panel, and Supplementary movie M2).

To determine whether extracellular matrix signal is required for the effect of NGAL on cell movement, we also performed time-lapse live-cell imaging analysis for the NGAL-overexpressing cells using uncoated glass slides. In contrast to that observed on collagen-coated slides, NGALoverexpressing cells had a similar phenotype with NGALunderexpressing cells, showing a tight compact growth pattern and restricted cell detachment from the cell colonies (Figure 3a, lower panel, and Supplementary movie M3). These observations suggest that NGAL promoted cell motility and detachment from cell colonies only in the presence of abundant extracellular matrix signal.

On account of the increased freedom of movement of the NGAL-overexpressing cells, we next investigated whether the invasive abilities of the cells were altered by performing in vitro invasion assays using type-IV collagen-coated transwell filters. We observed a significantly increased number of invading NGAL-overexpressing cells as compared with parental cells (Figure $3 \mathrm{~b}, P<0.001$ ). On the other hand, there were significantly fewer invading NGAL-underexpressing cells as compared with parental cells (Figure $3 \mathrm{~b}, P<0.05$ ). No significant difference in the invading cell numbers was observed between vector-transfected and parental cells (Figure 3b).

To further confirm the function of NGAL in promoting cell invasion ability, we chose two other colon cancer cell lines, DLD1 and HCT116, which showed relatively high levels 
of endogenous NGAL and E-cadherin (data not shown). We carried out knockdown experiments using siRNA for NGAL. After $48 \mathrm{~h}$ of siRNA or control siRNA transfection, the invasion assay was performed using collagen-IV-coated transwell filters. Twenty-four hours after incubation in the invasion chamber, we observed significantly decreased invading cell numbers from the NGAL knockdown cells compared with those from the control siRNA-transfected cells in both cell lines (Figure 3c). Cell lysates from the duplicated experiments were collected $72 \mathrm{~h}$ post-transfection of NGAL siRNA or control siRNA and subjected for western blotting analysis. We observed a decreased NGAL protein level in the NGAL siRNA-transfected cells compared with the control siRNA-transfected cells (Figure 3d).

For the tumor cells to become invasive, they must penetrate the basement membrane. MMPs are known to degrade most of the molecular components of the basement membrane, and MMP expression is a hallmark of cancer progression, including local invasion and distant metastasis. We first hypothesized that the increased cell-invasive activity induced by NGAL might result from the increased MMP9 levels in the cancer cells because (1) increased MMP9 levels have been reported in colon cancers; ${ }^{18,19}$ (2) NGAL and MMP9 have been reported to form a complex for increased MMP9 level and activity; ${ }^{2}$ and (3) increased levels of this complex have been observed in the urine of breast and bladder cancer patients. ${ }^{5,8}$ To assess this possibility, we first performed a western blot analysis to quantify the MMP9 protein levels in both conditioned media and cell lysates. A commercially available MMP9/NGAL complex served as a positive control. Surprisingly, except the positive control, we could not detect MMP9 protein in any of the cell lines (from either conditioned media or cell extract) using an
anti-MMP9 antibody in western blot analysis (Supplementary Figure S1A, panels a and b). However, NGAL protein was readily detectable in the conditioned media on the same blot (Supplementary Figure S1A, panel c).

To confirm the results from the western blot analysis, we next performed gelatin zymographic assays using the conditioned media from different cell lines. Results from the gelatin zymographic assay also revealed no detectable MMP9 activity in all the cell lines (Supplementary Figure S1B). We did observe some high-molecular-weight and low-molecularweight gelatinase activities in the conditioned media. However, the activity levels of these undetermined gelatinases were decreased in the NGAL-overexpressing cells (N11 and N15) and increased in the NGAL-underexpressing cells (N19 and N22) (Supplementary Figure S1B). These data suggest that for KM12C colon carcinoma cells, NGAL overexpression promoted the cell invasion ability through an MMP9-independent mechanism. Our observation of the decreased level of gelatinase activities from some unidentified highand low-molecular-weight species present in NGAL-overexpressing cells also suggests that the increased invasion we observed in NGAL-overexpressing cells was not due to the increased gelatinase activities.

\section{NGAL Overexpression Alters the Subcellular Localization of E-Cadherin/Catenin Proteins}

The proper formation of adhesion junctions, which are regions of the plasma membrane where E-cadherin molecules of adjacent epithelial cells contact each other, is critical in the maintenance of epithelial differentiation. ${ }^{20}$ To form tight intercellular adherens junctions, E-cadherin binds with $\beta$-catenin to form a complex and $\alpha$-catenin links the complex to actin cytoskeleton through binding with $\beta$-catenin. ${ }^{21}$

Figure 2 NGAL overexpression altered cytoskeletal organization and increased cell-matrix adhesion in KM12C colon carcinoma cells. (a) Analysis of NGAL expression by immunoblotting in parental $(\mathrm{P})$, empty vector-transfected control $(\mathrm{V})$, and NGAL stable clones transfected with NGAL/pDsRed-N2 containing antisense sequence (N19 and N22) and NGAL/pcDNA3.1(+) containing sense sequence (N11 and N15), respectively. A purchased NGAL/MMP9 protein was used as a positive control $(+)$. $\beta$-Actin levels were also monitored to normalize protein loading. The empty vector-transfected control cells show a level of NGAL expression similar to that of the parental cells. However, compared with the parental and vector-transfected control cells, the N19 and N22 clones show decreased expression in NGAL (downward arrow), whereas the N11 and N15 clones showed overexpression of NGAL (upward arrow). (b) Phasecontrast images of collagen-IV-adherent vector-transfected, NGAL-underexpressing (downward arrow, N22), and NGAL-overexpressing (upward arrow, N11) cells after $24 \mathrm{~h}$ of incubation. The images were taken with a ZEISS HAL 100 microscope at a magnification of $\times 200$. Scale bar: $50 \mu$ m. The vector-transfected cells are tightly compact, with several cell layers in the center, whereas the NGAL-underexpressing cells are smaller in size and show a more aggregated cellular growth pattern compared with the vector transfectants. In contrast, the NGAL-overexpressing cells have a more angular and flat morphology with increased spreading of peripheral cells and cellular cytoplasmic projections compared with the vector transfectants (arrowheads, right panel). In addition, the cells are larger in size compared with the vector control cells. (c) Morphological analysis of the cytoskeletal organization of collagen-IV-adherent NGALtransfected cells. The cells were incubated for $24 \mathrm{~h}$ and then fixed and stained with phalloidin-TR for F-actin and anti- $\alpha$-tubulin antibody for tubulin,

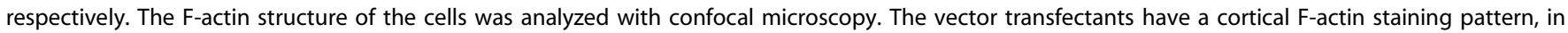
which F-actin is concentrated at the cell-cell junctions and at the edges of the cells in contact with the substratum (upper left panel). The NGAL-

underexpressing cells show a similar F-actin staining pattern as that of the vector transfectant, except that the cells are smaller (center panel). In contrast, the NGAL-overexpressing cells are larger and F-actin is located diffusely within the cells and at the edges of the cytoplasmic projections (upper right panel, arrows). The microtubule structure of the cells was analyzed by phase-contrast microscopy. A weak microtubule structure is evident in the vectortransfected and NGAL-underexpressing cells (lower left and center panels). In contrast, as is the case for F-actin staining, the NGAL-overexpressing cells are larger and exhibit prominent microtubules extending from the cell centers to the edges. The gray images are shown, and the scale bar is $50 \mu \mathrm{m}$. (d) Quantitation data from the cell-attachment assay. The cell-attachment assay was carried out as described in Materials and methods and the bar graph shows the percentage of attached cells among the total cells seeded, with the values in parentheses representing the standard deviation of triplicate

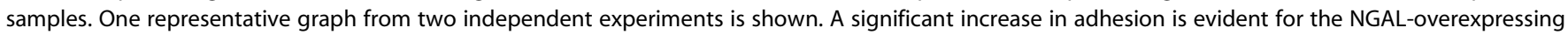
clones (N11 and N15; $P<0.001)$. 
b
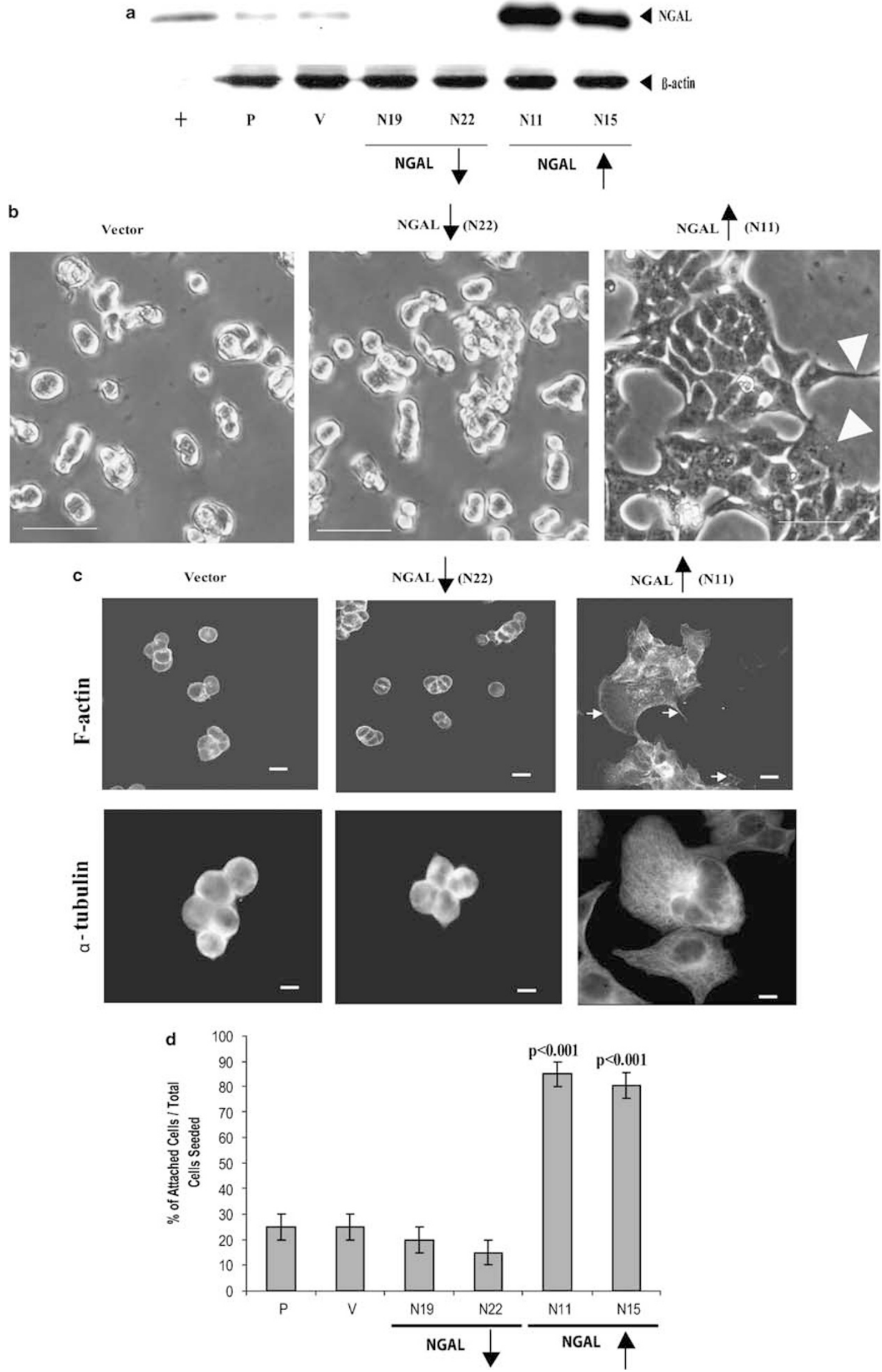
Loosening of these junctions has been reported to increase cell motility and contribute to invasion and metastasis of tumor epithelial cells. ${ }^{22-25}$ As we observed that NGAL-overexpressing cells showed reduced cell-cell adhesion and increased cell detachment from the epithelial colonies, we hypothesized that NGAL altered E-caherin/catenin complex. We first examined whether NGAL overexpression altered the expression of $\mathrm{E}$-cadherin, $\beta$-catenin, and $\alpha$-catenin. Western blot analyses from several repeated experiments using cell lysates extracted from the collagen-IV-adherent cells $24 \mathrm{~h}$ after seeding showed only minimal changes in the levels of these proteins in response to NGAL overexpression (Figure 4a).

We next performed immunofluorescence staining experiments to evaluate the subcellular localization of these molecules. We first visualized E-cadherin and $\beta$-catenin in the NGAL-overexpressing and the vector control cells cultured on routine glass slides for $24 \mathrm{~h}$. We observed that E-cadherin and $\beta$-catenin co-localized at cell-cell junctions in both cell lines (Supplementary Figure S2A). We then performed immunofluorescence staining experiments for these proteins on cells grown on type-IV collagen-coated glass slides. We observed that E-cadherin was localized mainly in the cell-cell junctions to form a honeycomb pattern in control cells (Figure $4 \mathrm{~b}$, upper panel), but showed a diffuse cytoplasmic staining pattern in NGAL-overexpressing cells (Figure 4b, lower panel). Similarly, $\beta$-catenin was found co-localized with E-cadherin at the cell-cell junctions in the control cells. By contrast, in the NGAL-overexpressing cells, a fraction of $\beta$-catenin appeared diffusely distributed in the cytoplasm (Figure $4 \mathrm{~b}$, lower panel, arrowheads). But unlike E-cadherin in these cells, a detectable amount of $\beta$-catenin remained at cell-cell contact sites. Thus, there was a dissociation of $\beta$ catenin from E-cadherin (Figure $4 \mathrm{~b}$, lower panel, arrows). Immunofluorescence staining studies for E-cadherin and $\beta$ catenin were also performed on collagen-IV-adherent KM12C parental cells and NGAL-underexpressing cells, with results very similar to those for the vector-transfected cells (Supplementary Figure S2B).

We next evaluated the subcellular localization of $\alpha$-catenin. As shown in Figure $4 c, \alpha$-catenin mainly localized along the cell-cell junction regions in the vector-transfected cells (Figure 4c, upper panel, arrows), whereas it showed a more diffuse pattern of staining in both the cytoplasm and nucleus in the NGAL-overexpressing cells (Figure 4c, lower panel, arrows). To confirm this observation, we carried out western blot analysis using nuclear and cytoplasmic cellular fractions extracted from NGAL-overexpressing and -underexpressing cells, as well as vector-transfected cells cultured on collagenIV dishes. As shown in Figure 4d, NGAL overexpression markedly increased the level of nuclear $\alpha$-catenin as compared with the NGAL-underexpressing and the vectortransfected cells. This result supports the earlier report in which $\alpha$-catenin was detected in cell nuclei from both colon carcinoma cell lines and tissues. ${ }^{26}$ Taken together, these studies show that NGAL overexpression, coupled with collagenIV signal, can serve as a spatial regulator of E-cadherin, $\beta$ catenin, and $\alpha$-catenin.

\section{NGAL Overexpression Decreases E-Cadherin-Mediated Cell-Cell Adhesion Through Rac1}

We sought to understand the mechanism by which NGAL modulates E-cadherin/catenin, cytoskeletal reorganization, and cell migration, and we focused our investigation on Racl. Racl is a member of the Rho small GTPase family and has been implicated in the establishment and maintenance of Ecadherin-mediated cell-cell adhesion as well as in the invasion and migration of epithelial tumor cells. ${ }^{27} \mathrm{Racl}$ is also a key regulator of cell polarization, actin and microtubule organization, and formation of lamellipodia and membrane ruffles. ${ }^{20,28-32}$ Abundant Rac1 has been visualized in migrating fibroblasts, with the highest concentrations at the leading edge. ${ }^{33-35}$ Inhibition of Rac1 caused the formation of a thick actin cortex at the cell periphery and inhibited the migration of intestinal epithelial cells. ${ }^{36}$ On the basis of the similarity in the cellular phenotypes induced by Racl and NGAL, we hypothesized that Rac1 is a mediator of NGAL effects.

As the functions of small GTPases in epithelial cells are known to be dependent on extracellular matrix signals, ${ }^{37}$ we carried out experiments with cells cultured on collagen-IVcoated plates or glass slides. We first evaluated Racl protein

Figure 3 NGAL overexpression increased KM12C colon carcinoma cell motility and in vitro invasion. (a) Time-lapse live-cell imaging. Frames from several time points are shown. The moving cells are indicated by arrow(s). The NGAL-underexpressing cells form tight clusters, and the cell (indicated by an arrow) at the margin of the colony shows dynamic membrane activity, with extension and retraction of finger-like projections (upper panel). In contrast, the NGALoverexpressing cells form loose and flatten colonies, and the cells (indicated by an arrow) migrate extensively across the surface. The cells show broken cellcell adhesion junctions (arrowheads, center panel). (b) In vitro invasion assay on collagen-IV filters. The invasion assays were performed as described in Materials and methods. The bar graph shows the number of invading cells on the lower side of the filters. The graph represents data from two independent experiments performed in triplicate. Significantly more NGAL-overexpressing cells (upward arrow) show invasion compared with the parental cells $(P<0.001$ and $P<0.02$ ), respectively. On the other hand, significantly fewer NGAL-underexpressing cells (downward arrow) show invasion compared with the parental cells $(P<0.05)$. No significant difference in invading cell number is observed between parental cells and vector-transfected controls. (c) In vitro invasion assay of DLD1 and HCT116 cells after NGAL siRNA or control siRNA transfection. The invasion assay was performed as described in Materials and methods, and the bar graph shows the number of invading cells on the lower side of collagen-IV-coated filters. The graph represents data from triplicate filters. Significantly more invading control siRNA-transfected cells are observed compared with NGAL siRNA-transfected cells for both DLD1 and HCT116 cell lines $(P<0.0003$ and $P<0.0001$, respectively). (d) Western blot analysis of NGAL levels in DLD1 and HCT116 cells $72 \mathrm{~h}$ post-NGAL siRNA and control siRNA transfection. A significantly lower NGAL level is shown in NGAL siRNA-transfected cells compared with the control siRNA-transfected cells in both cell lines. 
a
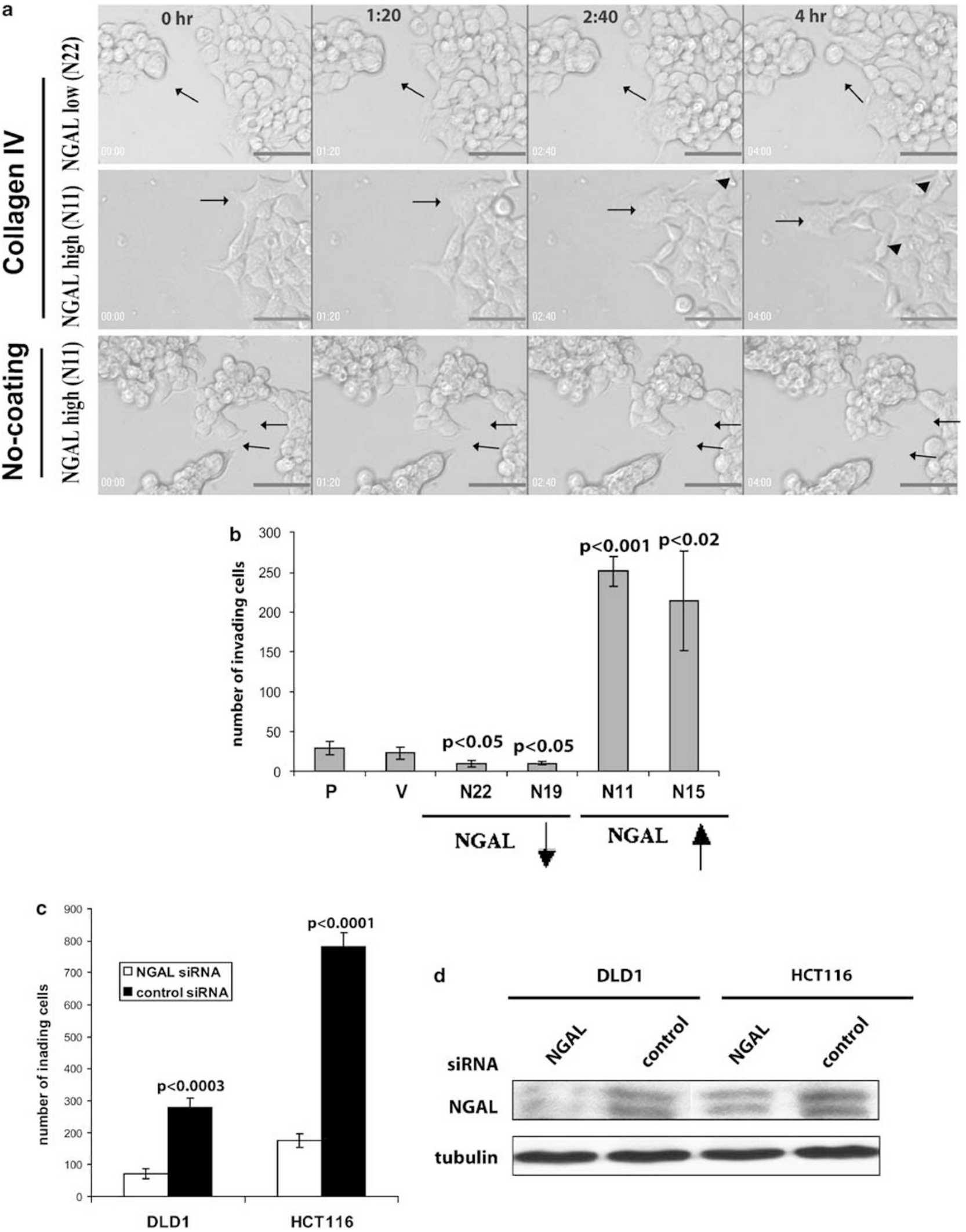

d

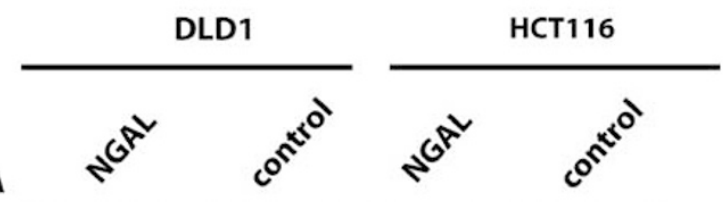

NGAL

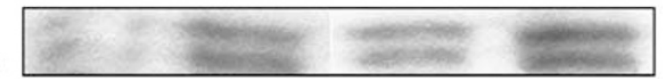

tubulin

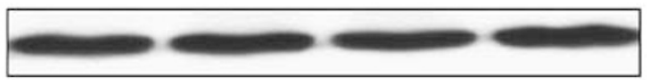


levels by western blot analysis but detected no significant changes at the protein levels among the different cell lines (Figure 5a). We next performed GTP-Racl pull-down assay to examine the levels of active GTP-Racl among the different cell lines. The GTP-Racl levels in the NGAL-overexpressing cells were only minimally increased compared with the parental, vector, and NGAL-underexpressing cell lines (data not shown). We then examined Racl subcellular localization by immunofluorescence staining. In vector control cells, Rac1 staining concentrated at the cell-cell junctions (Figure 5b, upper panel, arrows). In contrast, in the NGAL-overexpressing cells, Racl became relocalized to the cytoplasm and more Racl staining was detected in the leading-edge regions of lamellipodia (Figure 5b, lower panel, arrows). These data suggest that NGAL overexpression results in the polarized localization of Rac1 in the leading edge of cell migration.

To further investigate whether NGAL overexpression induced changes in E-cadherin localization through Racl, we transfected a DN Racl-GFP into the NGAL-overexpressing cells. The dominant-negative Racl blocks the endogenous Racl through binding preferentially GDP rather than GTP and thereby inhibits the activation of endogenous Racl by titrating out its $\mathrm{GEF}^{31}$ We observed co-localization of Ecadherin with DN Rac1-GFP at cell-cell junctions in the DN Rac1-GFP-transfected cells (Figure $5 c$, upper panel, green cells pointed by arrows). In contrast, E-cadherin still showed a diffuse pattern in the untransfected cells (Figure $5 c$, upper panel, arrowheads). As a control, we also transiently transfected a CA Rac1-GFP construct into the NGAL-overexpressing cells. The GFP fluorescence in the CA Rac1-GFPtransfected cells mainly appeared on the membrane and on the leading-edge areas where the cells were in contact with the substratum (Figure $5 \mathrm{c}$, lower panel, arrows). E-cadherin still showed a diffuse pattern in the transfected cells (Figure $5 c$, lower panel, arrows) similar to that in untransfected cells (Figure 5c, lower panel, arrowheads). In addition, the CA Rac1-GFP-transfected cells were also more spread out than the untransfected cells (Figure 5c, lower panel). These observations suggest that the activity of Rac1 in NGAL-overexpressing cells has a direct impact on E-cadherin-mediated cell-cell adhesions and the migratory morphology of the KM12C colon carcinoma cells.

\section{NGAL Affects E-Cadherin and Rac1 Through an Iron-Dependent Mechanism}

Iron is required for many redox processes in all eukaryotic and most prokaryotic cells. ${ }^{38,39}$ Most cells acquire iron by capturing iron-loaded transferrin. ${ }^{40}$ However, the transferrin pathway is not essential for the delivery of iron to many tissues, including epithelia. ${ }^{41}$ NGAL and $24 \mathrm{p} 3$ (the mouse homolog of human NGAL) are reported to be iron-trafficking proteins. ${ }^{41,42}$ Yang et $a l^{41}$ reported that NGAL delivers iron into cells and is internalized into endosomes and recycled. To investigate whether iron levels in cancer cells have a direct impact on NGAL effect, we treated different cell lines with either ferric chloride $(50 \mu \mathrm{M})$ or the iron chelator DFO $(20 \mu \mathrm{M})$. We first examined iron uploading by measuring cellular levels of ferritin, which is a major iron-storage protein in most cell types and positively regulated by cellular iron levels. ${ }^{41}$ Western blot analysis showed an increase in the levels of ferritin protein in NGAL-overexpressing cells (N11 and N15) but a decrease in NGAL-underexpressing cells (N19 and N22) compared with the parental cells after ferric chloride treatment (Figure 6a). No significant difference in the ferritin protein level was observed between parental and vector-transfected cells after the treatment (Figure 6a). In contrast, ferritin became undetectable in all cell lines after DFO treatment (data not shown). These observations showed that NGAL overexpression indeed led to cellular iron upload in iron-rich environment.

We next examined whether iron uploading by NGAL has a direct impact on E-cadherin. Western blot analysis showed that neither iron nor iron chelator altered the E-cadherin protein level in NGAL-overexpressing cells as compared with that in control cells (Figure 6b). We then visualized

Figure 4 NGAL overexpression altered E-cadherin cellular localization in KM12C colon carcinoma cells. (a) Western blot analysis of E-cadherin, $\alpha$-catenin, and $\beta$-catenin protein levels in collagen-IV-adherent cells. Whole-cell lysates extracted from parental, empty vector-transfected control, and NGALtransfected cells were subjected to western blot analysis. P: parental; V: empty vector-transfected control cells; N19 and N22: NGAL-underexpressing cells (downward arrow); N11 and N15: NGAL-overexpressing cells (upward arrow). For the proteins tested, no significant differences are evident among the different cell lines. The blot was re-probed with $\beta$-actin to normalize for protein loading. (b) Morphological analysis of cellular localization of endogenous Ecadherin and $\beta$-catenin in the collagen-IV-adherent vector-transfected and NGAL-overexpressing cells (N11). Endogenous E-cadherin and $\beta$-catenin were visualized by immunofluorescence staining using anti-E-cadherin-FITC and anti- $\beta$-catenin TRITC antibodies. In the vector control cells, E-cadherin and $\beta$ catenin are concentrated and co-localized at the cell-cell junctions (shown in yellow color, upper panel, arrows). However, the NGAL-overexpressing cells are more extended, and E-cadherin mainly assumes a diffuse pattern and is dissociated from $\beta$-catenin at the cell-cell junctions (lower panel, arrows). $\beta$ Catenin staining shows partial diffuse staining in the cytoplasm and partial staining in cell-cell borders (lower panel, arrowheads). The images were taken with a ZEISS HBO 100 phase-contrast microscope at a magnification of $\times 630$. Cell nuclei were counterstained with DAPI. Scale bar is $50 \mu$ m. (c) Localization of endogenous $\alpha$-catenin in the collagen-IV-adherent vector-transfected and NGAL-overexpressing cells (N11). The endogenous $\alpha$-catenin was visualized by double-fluorescence staining. In the vector-transfected cells, the $\alpha$-catenin concentrates at cell-cell junctions (upper panel), but a diffuse and nuclear localization pattern is seen in the NGAL-overexpressing cells (lower panel). Cell nuclei were counterstained with DAPI. Scale bar is $50 \mu \mathrm{m}$. (d) Western blot analysis of $\alpha$-catenin protein level using cytoplasmic $(\mathrm{C})$ and nuclear $(\mathrm{N})$ protein fractionations extracted from the vector-transfected cells $(\mathrm{V})$ and the NGALoverexpressing (N11) and NGAL-underexpressing cells (N22), respectively. The blot was re-probed with histone H1 and GAPDH to control for the quality of the fractionations and protein loading. 


\section{a}
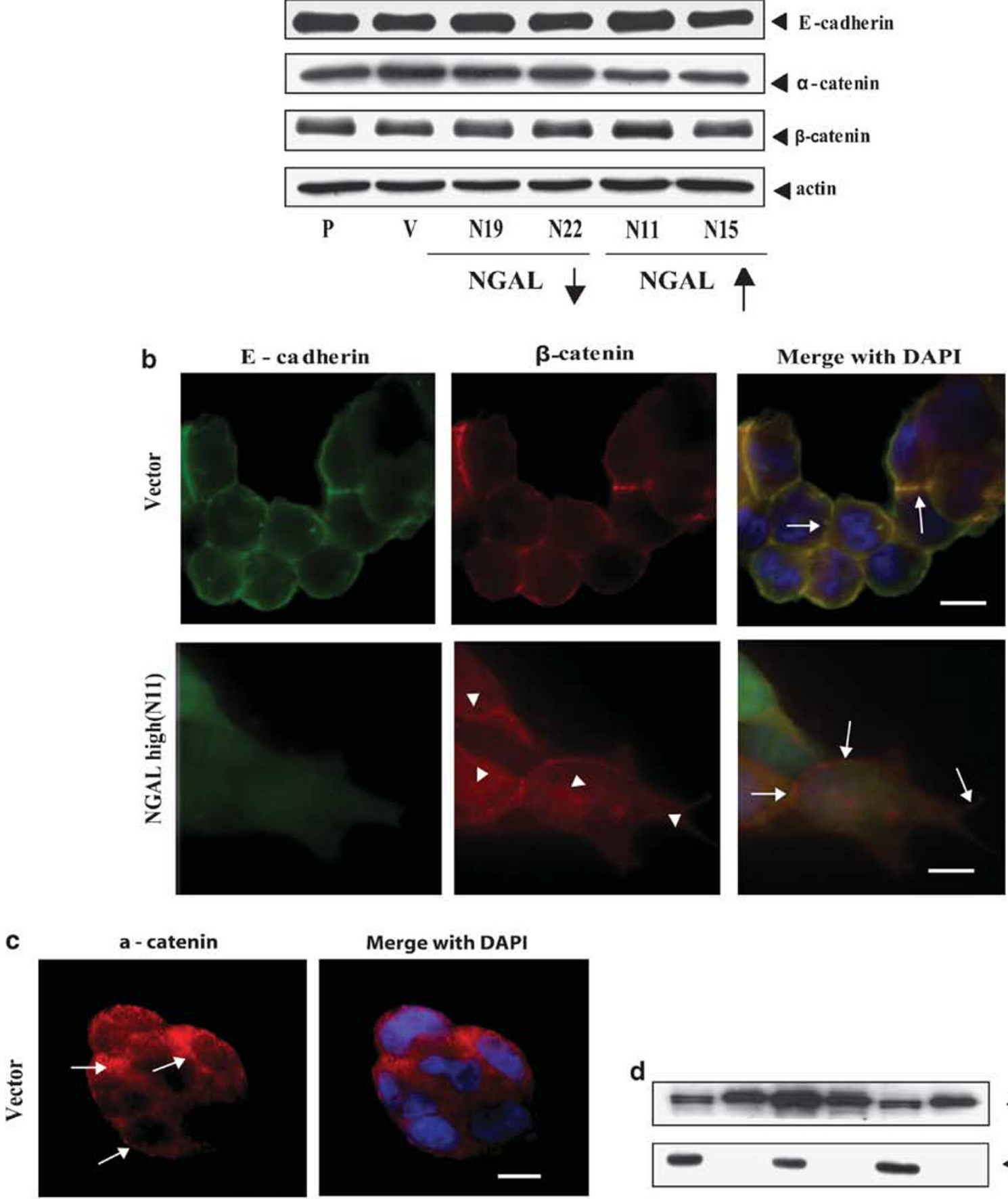

Merge with DAPI

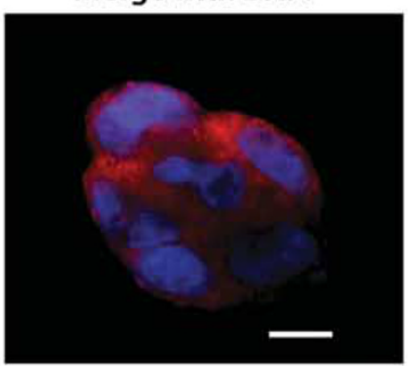

d
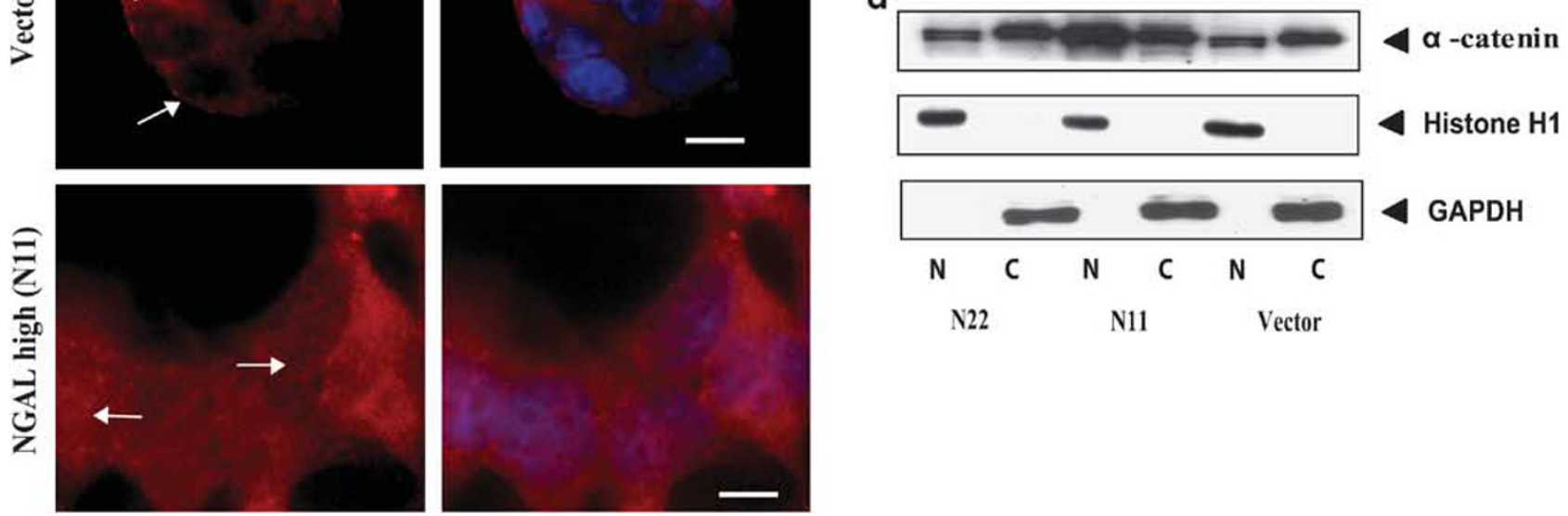
E-cadherin by immunofluorescence staining and examined its subcellular localization with fluorescence microscopy. As shown in Figure 6c, treatment with DFO for $24 \mathrm{~h}$ restored E-cadherin to the cell-cell junctions as compared with the untreated cells (Figure 6c, right panel, arrows). In addition, the DFO-treated cells showed a compact growth pattern similar to the parental cells. In contrast, treatment with ferric chloride for $24 \mathrm{~h}$ appeared to enhance the translocation of E-cadherin into the cytoplasm in the perinuclear regions (arrows), and the cells showed a loose cell-cell contact (arrowheads) as compared with the untreated cells (Figure 6c, left and center panels). Thus, our results indicate that the change in iron level in the NGAL-overexpressing cells had a direct impact on E-cadherin subcellular localization.

As we had shown that NGAL overexpression decreased E-cadherin-mediated cell-cell adhesions through Racl, we examined whether NGAL-mediated iron uploading is an upstream event for change in Racl activity. We therefore measured Racl activity by GTP-Racl pull-down assay after $24 \mathrm{~h}$ of ferric chloride $(50 \mu \mathrm{M})$ or DFO $(20 \mu \mathrm{M})$ exposure. However, we did not observe significant differences in GTP-Rac1 level in treated cells as compared with that in untreated cells (Figure 6d). We then visualized Rac1 by immunofluorescence staining and examined its subcellular localization in NGAL-overexpressing cells after $24 \mathrm{~h}$ of ferric chloride $(50 \mu \mathrm{M})$ or DFO $(20 \mu \mathrm{M})$ treatment. We observed enhanced Rac1 localization at the leading edge of the sheet-like lamellipodia in migrating cells (Figure 6e, center panel, arrows). In contrast, in the DFO-treated cells, Rac1 was partially diffuse in the cytoplasm (arrowheads) and partially localized on the peripheral membrane (arrows) (Figure 6e, right panel). These results suggest that NGAL alters Racl subcellular localization in an iron-dependent manner.

We next examined the effects of cellular iron uploading on NGAL-mediated cell invasion ability. We carried out an in vitro invasion assay using human collagen-IV-coated transwell filters and treated the NGAL-overexpressing cells with either ferric chloride $(50 \mu \mathrm{M})$ or DFO $(20 \mu \mathrm{M})$ for $24 \mathrm{~h}$. The results showed that ferric chloride treatment significantly increased the invading cell number $(P<0.0001)$. In contrast, DFO treatment significantly decreased the invading cell number $(P<0.01)$ as compared with the untreated control cells (Figure 6f). Thus, the iron levels in the cells had direct impact on the invasive capability of the cell.

To evaluate whether iron uploading also affects NGAL cellular distribution, we performed immunofluorescence staining to visualize the subcellular distribution of NGAL after ferric chloride or DFO treatment. NGAL showed a punctuate distribution pattern in the cytoplasm, with some particle structures in the untreated cells (Figure 6g, left panel, arrows). After treatment with $50 \mu \mathrm{M}$ ferric chloride for $24 \mathrm{~h}$, we observed a significant increase in the staining of NGAL particle structures especially in the cells at the leading edge of the cell colonies (Figure 6g, center panel, arrows). The NGAL staining in the cells behind the leading moving cells showed a much lower fluorescence intensity (Figure 6g, center panel, arrowheads). In contrast, after $24 \mathrm{~h}$ of treatment with $20 \mu \mathrm{M}$ DFO, we observed a diffused pattern of NGAL fluorescence in the cytoplasm (Figure 6g, right panel, arrows). The NGALpositive punctuated particles in the cells are likely secretary vesicles. If it is true, this may indicate that increasing iron level in cell environment might promote cellular trafficking of NGAL. To examine whether iron level affects NGAL secretion, we performed western blot analysis to examine NGAL level in both cell lysates and the conditioned medium after ferric chloride or DFO treatment. As expected, a higher level of NGAL in the conditioned medium and a lower level of NGAL in the cell lysate from the ferric chloride-treated cells were observed when compared with untreated cells (Figure 6h). Opposite results were observed in DFO-treated cells (Figure 6h). Thus, these data indicated that iron uploading in the cells promoted NGAL cellular trafficking. To confirm the effects observed in ferric chloride and DFO treatments, we also examined ferritin protein levels in the cells by western blot using cell lysates extracted from the treated and untreated control cells. As shown in Figure 6h, ferric chloride treatment significantly increased cellular ferritin levels, whereas the protein became undetectable after DFO treatment (Figure 6h).

In addition, we observed that an extended treatment (up to $48 \mathrm{~h}$ ) with ferric chloride caused moderate cell death and detachment from the plates in parental and vector control cells and severe cell death and detachment in

Figure 5 NGAL overexpression altered Rac1 subcellular localization. (a) Western blot analysis of Rac1 expression. Whole-cell lysates from collagen-IV-adherent cells were subjected to western blot analysis using anti-Rac1 antibody. The blot was re-probed with anti- $\beta$-actin antibody to normalize for protein loading. (b) Visualization of Rac1 protein in the vector-transfected and the NGAL-overexpressing cells. The cells were incubated on a collagen-IV-coated glass slide, fixed, stained with anti-Rac1-FITC antibody and analyzed by phase-contrast microscopy. Rac1 is evident at cell-cell junctions in the vector-transfected cells (upper panel, arrows), but in contrast, it shows a diffuse pattern in the cytoplasm and at the leading edges of the lamellipodia in contact with the substratum in the NGAL-overexpressing cells (lower panel, arrows). Cell nuclei were counterstained with DAPI. Scale bar is $50 \mu \mathrm{m}$. (c) Effects of DN Rac1-GFP and CA Rac1-GFP on E-cadherin subcellular localization. DN Rac1-GFP and CA Rac1-GFP constructs were transiently transfected into NGAL-overexpressing cells cultured on collagenIV-coated glass slide. At $48 \mathrm{~h}$ post-transfection, the cells were fixed and the endogenous E-cadherin was double stained (red). DN Rac1-GFP restored E-cadherin to the cell-cell junctions, and the two proteins show co-localization at the cell-cell borders (upper panel, arrows). By contrast, E-cadherin shows a diffuse staining pattern in the non-transfected cells (upper panel, arrowheads). In contrast, the CA Rac1-GFP is evident on the cell membrane and at the edge in contact with the substratum (lower panel, arrows). E-Cadherin shows an even more diffuse pattern (lower panel, arrows on red) as compared with the non-transfected cells (lower panel, arrowheads). Cell nuclei were counterstained with DAPI. Scale bar is $50 \mu \mathrm{m}$. 
NGAL-underexpressing cells. In contrast, the NGAL-overexpressing cells were resistant to the iron treatment and showed healthy morphology after a prolonged ferric chloride treatment (data not shown).

\section{DISCUSSION}

NGAL has recently been shown to be elevated in both leukemia cells and a wide spectrum of solid tumor cells. Whereas the mouse counterpart of NGAL, $24 \mathrm{p} 3$, has been suggested to
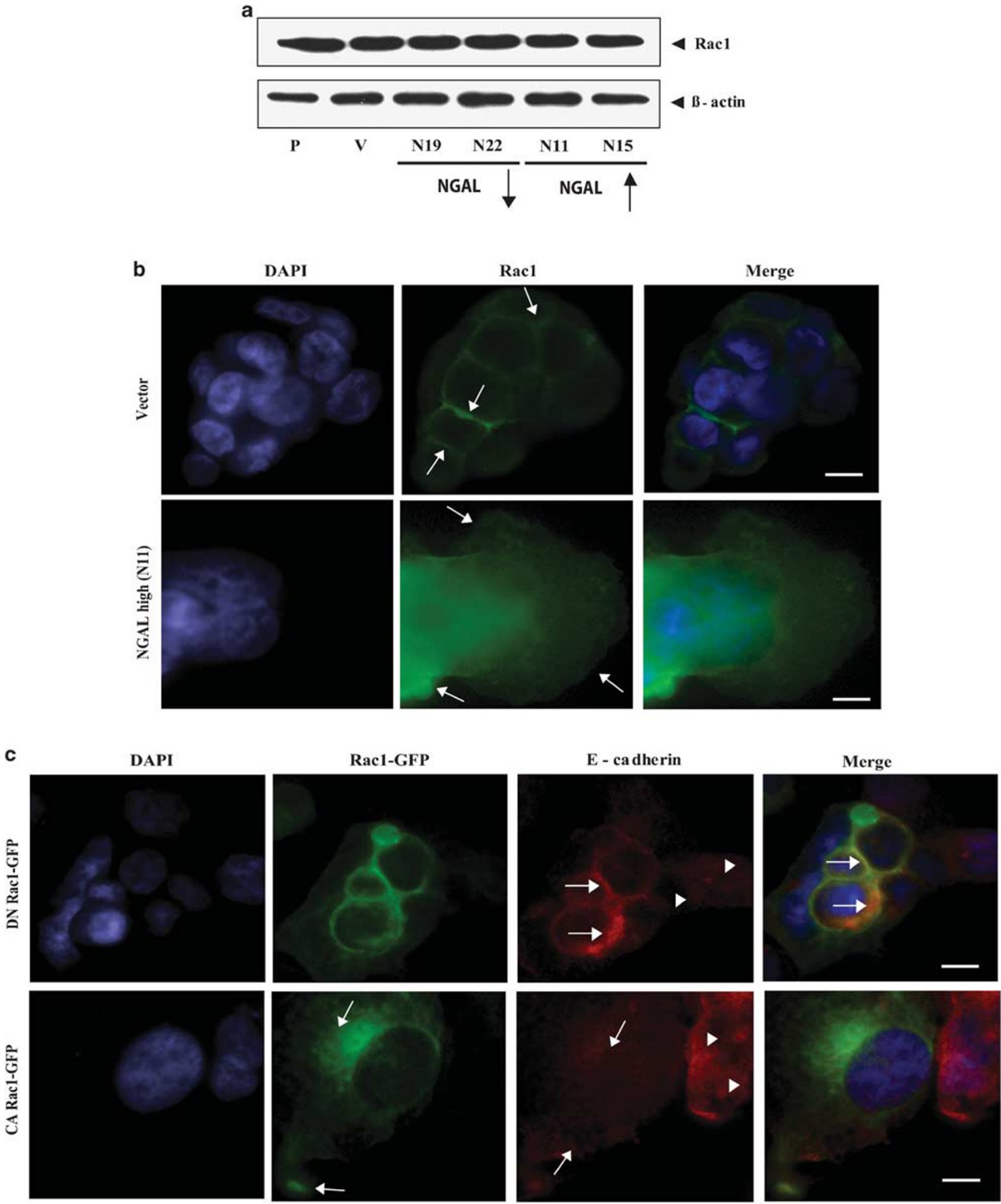
induce cell death of normal lymphocytes to provide leukemia cells in the vicinity with growth advantage, ${ }^{43}$ this mechanism is not apparent in solid tumors because the NGAL expressed in solid tumor cells has not been shown to induce cell death of co-incubated lymphocytes ${ }^{44,45}$ (and our unpublished work). We believe that the current study provides an important insight into the role of NGAL in solid tumor pathophysiology. NGAL, together with integrating extracellular matrix cues and iron, functions to control cell-cell and cellmatrix interactions by regulating Racl activity, thus modulating intracellular localization of the E-cadherin/catenin complex and cytoskeletal structure. Most importantly, this study revealed a complex relationship between tumor cells and their microenvironment. Specifically, in the presence of pure collagen IV in our in vitro system, iron enhanced the effect of NGAL on translocating E-cadherin/catenin subcellular localization from cell-cell junction and on enhancing cell invasion. Removal of iron by a chelator had an opposite effect. By contrast, on uncoated culture plates, the effect of NGAL was not apparent.

As NGAL is known to form a covalent interaction with MMP9, which promotes invasive behavior, ${ }^{2}$ we initially hypothesized that NGAL might promote cell invasion through increasing MMP9 activity. However, we did not detect MMP9 in either the cell lysate or the conditioned medium from our NGAL-overexpressing cells using an anti-human MMP9 antibody. Therefore, the phenotype we observed in this study is independent of MMP9, although the presence of MMP9 may further enhance the role of NGAL in cell invasion. We did observe some high- and low-molecular-weight gelatinase activities in the conditioned media, but the activity levels of these undetermined gelatinases were decreased in NGAL-overexpressing cells. Thus, increased gelatinase activity is not necessarily responsible for the role of NGAL in promoting invasion of colon carcinoma cells.

\section{NGAL and Cellular Community Effects}

Cell-cell and cell-matrix adhesion structures play a very important role in differentiated tissues and in cell migration associated with development, tissue remodeling, and pathological states. ${ }^{46}$ Strong cell-cell interactions result in a tight cell cluster as a community and constrain cells from moving away. A break of this community effect by dysregulation of E-cadherin/catenin complexes has been shown to be responsible for invasion and metastasis of cancer cells. ${ }^{23,24}$ Owing to the sequestration of E-cadherin from cell-cell junctions, the colon carcinoma cells were less adhesive to the cell aggregates and more adhesive to the matrix protein and assumed a 'scattering' growth pattern. The in vitro invasion assays performed in our study confirmed that

Figure 6 Effects of iron and iron chelator DFO treatments on E-cadherin, Rac1, and NGAL cellular distribution. For all experiments, cells were cultured on collagen-IV-coated plates or glass slides and treated with ferric chloride $(50 \mu \mathrm{M})$ or iron chelator DFO $(20 \mu \mathrm{M})$ for $24 \mathrm{~h}$. (a) Western blot analysis of ferritin protein level in the cells after $24 \mathrm{~h}$ of ferric chloride $(50 \mu \mathrm{M})$ treatment. As compared with the parental cells (P), a higher ferritin level is evident in the NGALoverexpressing cells (N11 and N15, upward arrow), whereas a lower ferritin level is seen in the NGAL-underexpressing cells (N19 and N22, downward arrow), and no significant difference is evident between the parental cells $(\mathrm{P})$ and the vector-transfected cells $(\mathrm{V})$. $\beta$-Actin was used to control for the protein loading. (b) Western blot analysis of E-cadherin. Whole-cell lysates extracted from parental (P), empty vector-transfected cells (V), NGAL-underexpressing cells (N19 and N22, downward arrow), and NGAL-overexpressing cells (N11 and N15, upward arrow) were subjected to western blot analysis. No significant differences are evident among the different cell lines. The blot was re-probed with $\beta$-actin to normalize for protein loading. (c) Subcellular localization of $\mathrm{E}$ cadherin in NGAL-overexpressing cells. The cells were fixed and stained with anti-E-cadherin-FITC antibody. After ferric chloride treatment, E-cadherin shows a cytoplasmic and perinuclear localization pattern (center upper panel, arrows) as compared with the no-treatment control cells. Some cells break away from the colony (arrowheads, center lower panel). However, DFO treatment completely restored E-cadherin to the cell-cell junctions (right panel, arrows) and cells show a more compact growth pattern as compared with the no-treatment control cells. Cell nuclei were counterstained with DAPI. Scale bar is $50 \mu \mathrm{m}$. (d) GTP-Rac1 pull-down assay of NGAL-overexpressing cells. The GTP-Rac1 pull-down assay was performed as described in Materials and methods. No significant difference in GTP-Rac1 level is observed after ferric chloride or DFO treatment compared with the no-treatment control cells. (e) Localization of Rac1 in NGAL-overexpressing cells. The NGAL-overexpressing cells without treatment show a spreading growth pattern and Rac1 is mainly localized on the membranes, especially at the protrusion tips (arrows, upper left panel). However, after ferric chloride treatment, the leading-edge migrating cells develop huge lamellipodia and Rac1 is mainly localized on cell membranes, with the highest concentrations at the leading edge of the sheet-like lamellipodia (center panel, arrows) as compared with the no-treatment control cells. By contrast, after treatment with the iron chelator DFO, Rac1 shows a diffuse cytoplasmic staining pattern (right panel, arrowheads). Even though Rac1 is also concentrated on cell membranes in some margin cells (right panel, arrows), the cells have lost their polarity and show a compact growth pattern (right panel). The cell nuclei were counterstained with DAPI. Scale bar is $50 \mu$ m. (f) Effects of ferric chloride and iron chelator DFO on cell invasion ability. The NGAL-overexpressing cells were cultured on collagen-IV-coated transwell filters and treated with either ferric chloride $(50 \mu \mathrm{M})$ or iron chelator DFO $(20 \mu \mathrm{M})$ for $24 \mathrm{~h}$. The graph of data from one of the two representative experiments is shown. The values in parentheses represent standard deviations from triplicate samples. The number of invading cells is significantly higher in ferric chloride-treated cells than that from the cells with no treatment $(P<0.0001)$, but it is significantly lower in the DFO-treated cells $(P<0.01)$ than that from the control cells. (g) Effects of iron or DFO on NGAL subcellular localization. NGAL-overexpressing cells were double stained with an anti-NGAL antibody. In the untreated cells, NGAL shows a punctuated cytoplasmic distribution with some particle structures (arrows, left panel). After ferric chloride treatment, NGAL mainly shows in particle structures and these concentrate in the cytoplasm of the leading-edge migrating cells (arrows, center panel). NGAL shows an almost uniform and diffused pattern in the cells after the DFO treatment (arrows, right panel). (h) Western blot analysis of ferritin and NGAL protein levels in NGAL-overexpressing cells. Both the cell lysate and the conditioned media were subjected to western blot analysis. The lysate and the conditioned media from the no-treatment cells were used as control. The ferric chloride treatment significantly increases the ferritin protein level, whereas in contrast, the DFO treatment significantly decreases the protein in the cells compared with the cells without treatment. For NGAL, ferric chloride treatment increases NGAL level in the conditioned media, but decreases NGAL level in the cell lysate compared with that from the cells without treatment, whereas, the iron chelator (DFO) treatment shows an opposite results. The blot was re-probed with $\beta$-actin to normalize for protein loading. 


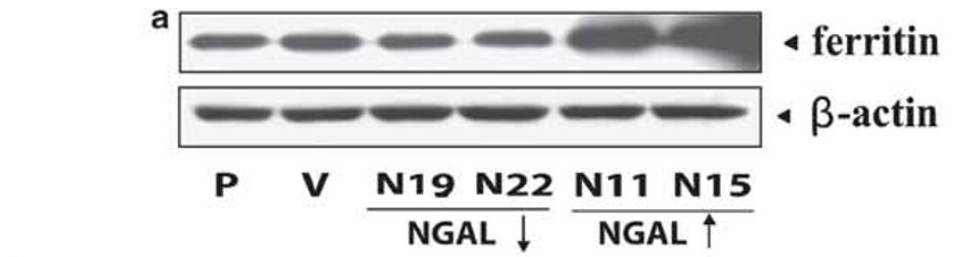

b
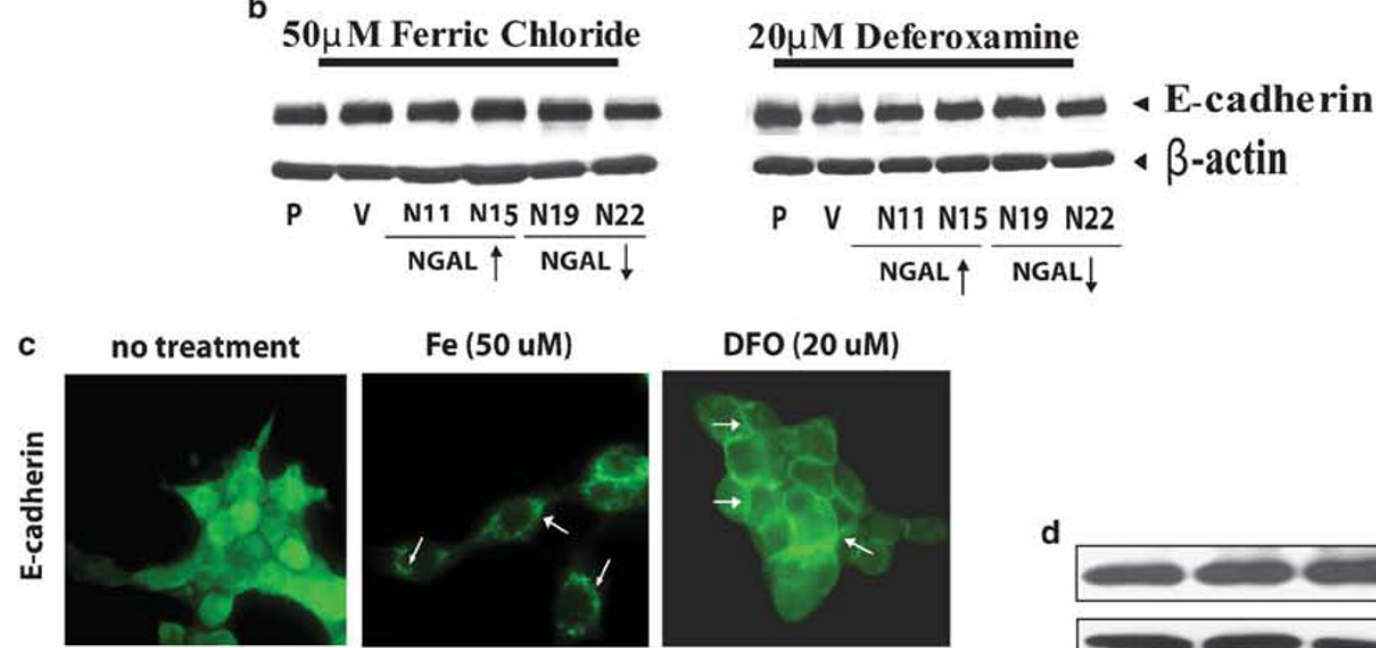

DFO (20 uM)
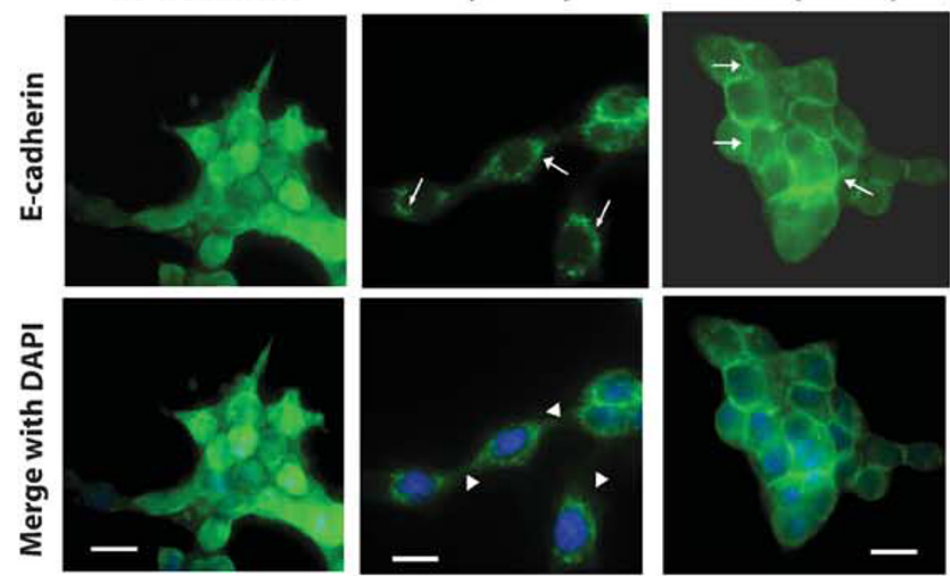

d
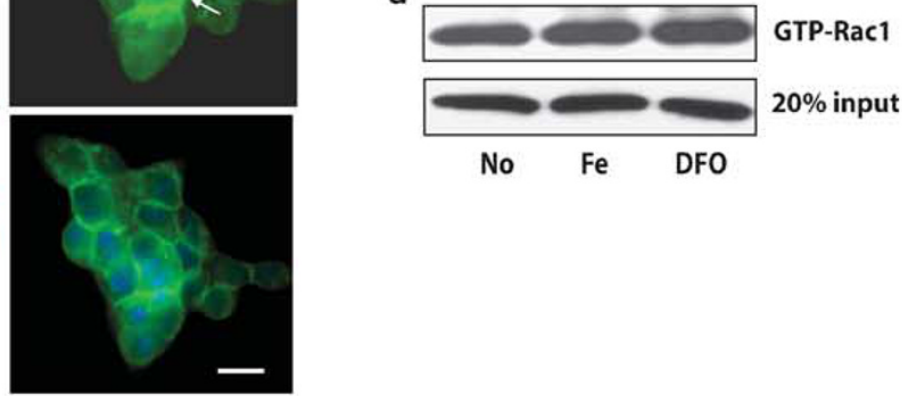

NGAL $\uparrow \frac{\text { N19 N22 }}{\text { NGAL } \downarrow}$
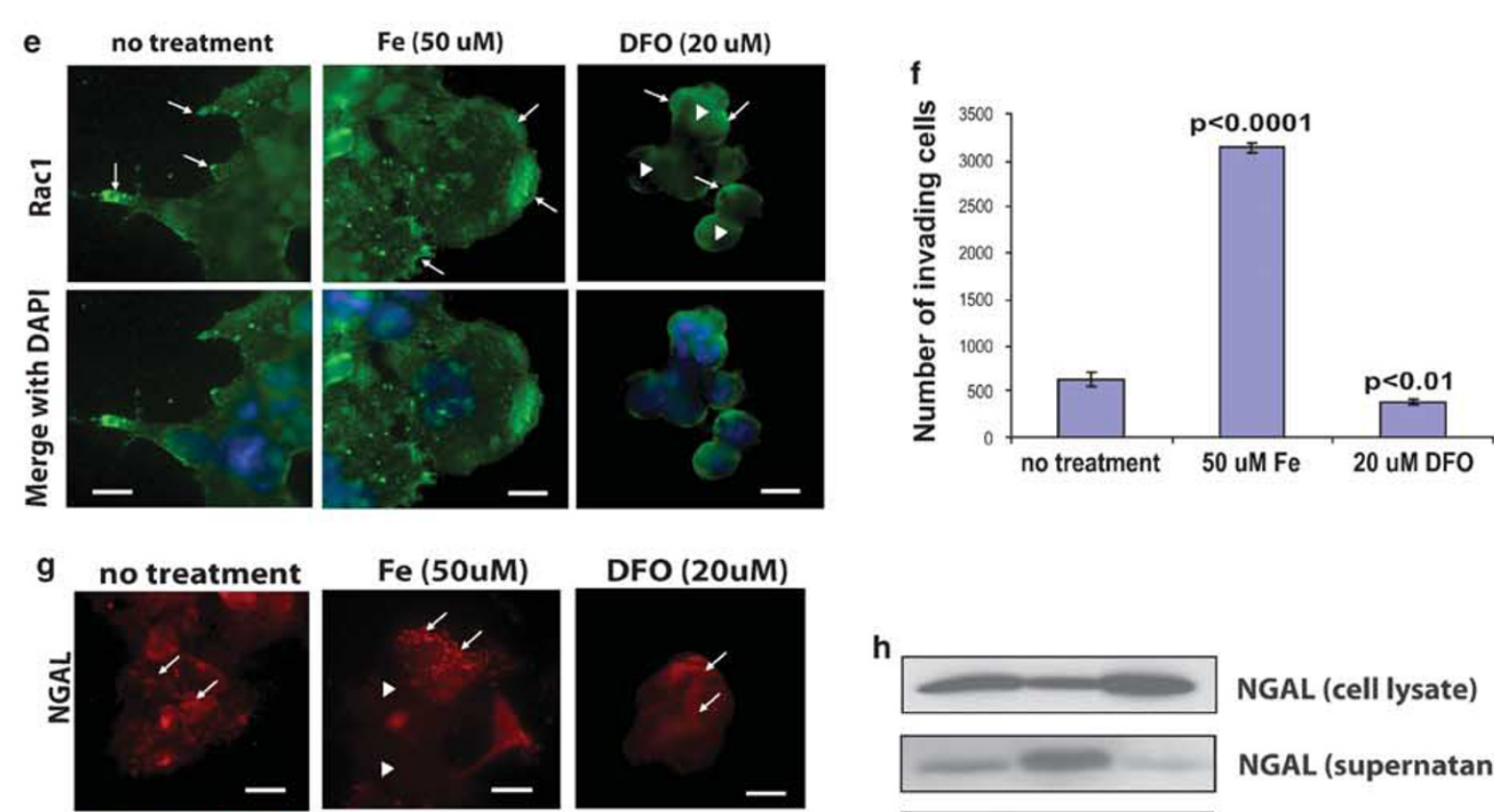

NGAL (cell lysate)
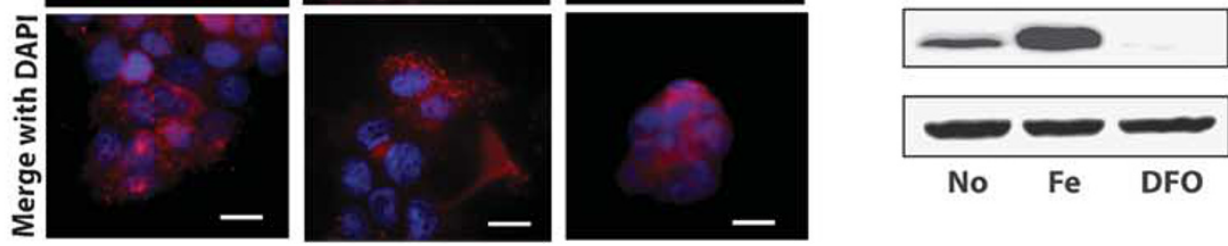

NGAL (supernatant)

ferritin

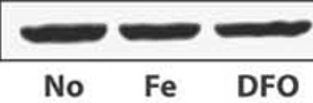

actin 
NGAL-overexpressing cells indeed were more invasive, and our time-lapse live-cell imaging clearly showed more freedom for NGAL-overexpressing cells to move. In contrast, the NGAL-underexpressing cells exhibited a more aggregated growth pattern. This is consistent with our understanding of the genesis of colorectal carcinoma. That is, the membrane localization of E-cadherin and catenin is critical for maintaining normal epithelial structure. However, in colorectal carcinoma and its precursors, the malignant glands are disorganized.

\section{Effects of NGAL on Rac1's Regulation of E-Cadherin}

The small Rho GTPases, including Rac1, cdc42, and RhoA, have been implicated in the establishment and maintenance of E-cadherin-mediated cell-cell adhesion as well as in the invasion and migration of epithelial tumor cells. ${ }^{28,29,34,47-50}$ The activities of Racl in epithelial cells are dependent on integrin signaling through interaction with extracellular matrix. ${ }^{37}$ In this study, we showed that NGAL overexpression decreased E-cadherin-mediated cell-cell adhesions on collagen-coated plates but not on uncoated plates. Therefore, we hypothesized that NGAL might regulate E-cadherin signaling through the modulation of Racl. We directly tested this hypothesis by the transfection of DN Rac1-GFP or CA Rac1GFP into the NGAL-overexpressing cells. Results from this experiment showed that DN Rac1-GFP restored E-cadherin to the cell-cell junctions (Figure 5c, upper panel), whereas CA Rac1-GFP further promoted E-cadherin cytoplasmic distribution and cell spreading (Figure $5 c$, lower panel). This experiment showed that Racl is a downstream mediator for the effect of NGAL on E-cadherin. Racl is also known to be one of the key regulators in cell polarization, actin organization, and cell migration. ${ }^{30-32}$ Racl has been reported to regulate the formation of lamellipodia and membrane ruffles, and active Racl can be visualized in migrating fibroblasts, with the highest concentrations at the leading edge. ${ }^{31,33,35}$ Inhibition of Racl activation causes the formation of a thick actin cortex at the cell periphery and inhibits migration of intestinal epithelial cells. ${ }^{36}$ In addition, Rac1 has also been reported to facilitate cell-matrix attachment. ${ }^{29}$ Our observation of Racl localization along the leading-edge region of the moving cells at the margin of the cell clusters in the NGAL-overexpressing cells further supports Racl as a mediator of NGAL's effect. Clearly, Racl in the leading migrating cells showed higher activity in which it showed higher fluorescence-staining intensity and concentrated at the leading edge of the lamellipodia, especially after ferric chloride treatment compared with the control cells (Figures $5 \mathrm{~b}$ and $6 \mathrm{e}$ ). The reason that we did not detect higher Racl activity from GTP-Racl pull-down assay among different cell lines might be the small population of the migrating cells in the cultured cells. At present, it is still not clear how NGAL affected the subcellular distribution and activity of Racl.

\section{Effects of Iron on NGAL Function}

Recently, it has been reported that NGAL is an iron-delivery molecule and can transport iron into cells. ${ }^{41,42,51}$ We confirmed that NGAL overexpression indeed increased cellular iron uploading in an iron-rich environment. We then directly tested whether iron levels in the cells could alter the cellular localization of Rac1 and E-cadherin by treating the NGALoverexpressing cells with DFO, an iron chelator. We showed that removal of iron by DFO restored E-cadherin to the cellcell junctions in the presence of type-IV collagen. DFO treatment also diminished polarized cellular morphology and inhibited cell invasion. However, DFO treatment did not alter E-cadherin protein levels in our experiment. In a recent study, E-cadherin and other paracellular junction proteins were decreased in iron-loaded hepatocytes, ${ }^{52}$ suggesting that iron loading may affect both E-cadherin subcellular localization and protein levels in the cells, depending on context. In contrast to DFO treatment, ferric chloride treatment further promoted cell spreading and E-cadherin cytoplasmic diffusion on collagen-IV-coated matrix. Further, Rac1 localized with the highest concentration at the leading edges of lamellipodia in moving cells upon ferric chloride treatment of NGAL-overexpressing cells. These results suggest that NGAL overexpression induces alteration of Rac1 cellular distribution through an iron-dependent mechanism. Again, this effect is dependent on the presence of collagen-IV matrix protein because we did not detect this effect on uncoated slides (data not shown).

The extracellular matrix-dependent and iron effects of NGAL may explain some controversial results with respect to the effect of NGAL on cell invasion. In the study of Hanai et $a l^{53}$, NGAL was found to diminish the invasiveness and metastasis of Ras-transformed cells. We first reasoned that one possible explanation was that $K$-ras mutation altered the function of NGAL. To rule out this possibility, we sequenced $\mathrm{K}$-ras in the KM12C cells used in our study and confirmed that there was no mutation (unpublished results). Another potential explanation for the differences in results was the cell type used in our studies. This possibility was suggested by the fact that malignant epithelial cells were used in both our study and a breast cancer study in which NGAL was similarly shown to enhance metastasis in an animal model. ${ }^{5}$ In contrast, Hanai et $a l^{53}$ used mesenchymal cells. It is also likely that different experimental conditions (cellular environment) may account for the differences. Recently, Lee et al ${ }^{54}$ reported that a C-terminal-tagged form of NGAL expressed in lentivirus inhibited the invasion of KM12SM cells, a metastatic clone of the KM12C cell line used in our study. Differences in the extracellular matrix environment used in their study may also be a cause of difference in the results.

In summary, our study showed that NGAL, which is elevated in majority of colon carcinoma tissues, contributes to colon cancer pathophysiology through the alteration of Rac1 cellular distribution and E-cadherin-mediated cell-cell adhesion. This regulation is dependent on extracellular 
matrix signals and iron levels in the cells, but is independent of MMP9.

Supplementary Information accompanies the paper on the Laboratory Investigation website (http://www.laboratoryinvestigation.org)

\section{ACKNOWLEDGEMENTS}

We thank Hong Zheng for her technical assistance. This study was partially supported by a grant from the National Foundation for Cancer Research, the Favrot Fund, the Wilson Fund, the Kadoorie Charitable Foundation, Cancer Center Support Grant P30 CA016672 from the National Cancer Institute, National Institutes of Health, and the Tobacco Settlement Fund to the MD Anderson Cancer Center as appropriated by the Texas State Legislature. Dr Hamilton is a recipient of the Frederick F Becker Distinguished University Chair in Cancer Research from The University of Texas. We thank Dr Randall Evans in the Image Analysis Core of MD Anderson Cancer Center for his help with confocal images; Dr Gregory Fuller and Lynda Corley in the Tissue Microarray Core for assistance with tissue microarray construction; and Dr Lucian Chirieac, Ellen Taylor, and Renee Webb for their technical support for immunohistochemistry studies. We also thank Ms Beth Notzon for editorial assistance.

1. Flower DR. The lipocalin protein family: structure and function. Biochem J 1996;318(Part 1):1-14.

2. Kjeldsen $L$, Johnsen $A H$, Sengelov $H$, et al. Isolation and primary structure of NGAL, a novel protein associated with human neutrophil gelatinase. J Biol Chem 1993;268:10425-10432.

3. Yan L, Borregaard N, Kjeldsen L, et al. The high molecular weight urinary matrix metalloproteinase (MMP) activity is a complex of gelatinase B/MMP-9 and neutrophil gelatinase-associated lipocalin (NGAL). Modulation of MMP-9 activity by NGAL. J Biol Chem 2001;276:37258-37265.

4. Yang J, Mori K, Li JY, et al. Iron, lipocalin, and kidney epithelia. Am J Physiol Renal Physiol 2003;285:F9-18.

5. Fernandez CA, Yan L, Louis G, et al. The matrix metalloproteinase-9/ neutrophil gelatinase-associated lipocalin complex plays a role in breast tumor growth and is present in the urine of breast cancer patients. Clin Cancer Res 2005;11:5390-5395.

6. Friedl A, Stoesz SP, Buckley P, et al. Neutrophil gelatinase-associated lipocalin in normal and neoplastic human tissues. Cell type-specific pattern of expression. Histochem J 1999;31:433-441.

7. Furutani $M$, Arii $S$, Mizumoto $M$, et al. Identification of a neutrophil gelatinase-associated lipocalin mRNA in human pancreatic cancers using a modified signal sequence trap method. Cancer Lett 1998;122:209-214.

8. Monier F, Surla A, Guillot $M$, et al. Gelatinase isoforms in urine from bladder cancer patients. Clin Chim Acta 2000;299:11-23.

9. Santin $A D$, Zhan F, Bellone $S$, et al. Gene expression profiles in primary ovarian serous papillary tumors and normal ovarian epithelium: identification of candidate molecular markers for ovarian cancer diagnosis and therapy. Int J Cancer 2004;112:14-25.

10. Stoesz SP, Friedl A, Haag JD, et al. Heterogeneous expression of the lipocalin NGAL in primary breast cancers. Int J Cancer 1998;79:565-572.

11. Lin $\mathrm{H}$, Monaco G, Sun T, et al. Bcr-Abl-mediated suppression of normal hematopoiesis in leukemia. Oncogene 2005;24:3246-3256.

12. Devireddy LR, Gazin C, Zhu X, et al. A cell-surface receptor for lipocalin $24 \mathrm{p} 3$ selectively mediates apoptosis and iron uptake.[see comment]. Cell 2005;123:1293-1305.

13. Wang $\mathrm{H}$, Wang $\mathrm{H}$, Shen $\mathrm{W}$, et al. Insulin-like growth factor binding protein 2 enhances glioblastoma invasion by activating invasionenhancing genes. Cancer Res 2003;63:4315-4321.

14. Nielsen BS, Borregaard N, Bundgaard JR, et al. Induction of NGAL synthesis in epithelial cells of human colorectal neoplasia and inflammatory bowel diseases. Gut 1996;38:414-420.

15. Bates RC, Mercurio AM. The epithelial-mesenchymal transition (EMT) and colorectal cancer progression. Cancer Biol Ther 2005;4:365-370.

16. Jawhari $A \cup$, Buda $A$, Jenkins $M$, et al. Fascin, an actin-bundling protein, modulates colonic epithelial cell invasiveness and differentiation in vitro. Am J Pathol 2003;162:69-80.
17. Danen $\mathrm{EH}$, van Rheenen J, Franken W, et al. Integrins control motile strategy through a Rho-cofilin pathway.[erratum appears in J Cell Biol. 2005 Aug 1;170(3):497]. J Cell Biol 2005;169:515-526.

18. Matsuyama Y, Takao S, Aikou T. Comparison of matrix metalloproteinase expression between primary tumors with or without liver metastasis in pancreatic and colorectal carcinomas. J Surg Oncol 2002;80:105-110.

19. Mook OR, Frederiks WM, Van Noorden CJ. The role of gelatinases in colorectal cancer progression and metastasis. Biochim Biophys Acta 2004;1705:69-89.

20. Evers $\mathrm{EE}$, Zondag GC, Malliri $\mathrm{A}$, et al. Rho family proteins in cell adhesion and cell migration. Eur J Cancer 2000;36:1269-1274.

21. Gooding JM, Yap KL, Ikura M. The cadherin-catenin complex as a focal point of cell adhesion and signalling: new insights from threedimensional structures. Bioessays 2004;26:497-511.

22. Birchmeier W, Behrens J. Cadherin expression in carcinomas: role in the formation of cell junctions and the prevention of invasiveness. Biochim Biophys Acta 1994;1198:11-26.

23. Birchmeier W, Hulsken J, Behrens J. Adherens junction proteins in tumour progression. Cancer Surv 1995;24:129-140.

24. Birchmeier W, Hulsken J, Behrens J. E-cadherin as an invasion suppressor. Ciba Found Symp 1995;189:124-136; discussion 36-41.

25. Bracke ME, Van Roy FM, Mareel MM. The E-cadherin/catenin complex in invasion and metastasis. Curr Top Microbiol Immunol 1996;213 (Part 1):123-161.

26. El-Bahrawy M, Talbot I, Poulsom R, et al. Variable nuclear localization of alpha-catenin in colorectal carcinoma. Lab Invest 2002;82:1167-1174.

27. Akhtar N, Hudson KR, Hotchin NA. Co-localization of Rac1 and E-cadherin in human epidermal keratinocytes. Cell Adhes Commun 2000;7:465-476.

28. Braga VM, Machesky LM, Hall A, et al. The small GTPases Rho and Rac are required for the establishment of cadherin-dependent cellcellcontacts. J Cell Biol 1997;137:1421-1431.

29. Fukata M, Nakagawa M, Kaibuchi K. Roles of Rho-family GTPases in cell polarisation and directional migration. Curr Opin Cell Biol 2003;15:590-597.

30. Hall A, Paterson HF, Adamson $P$, et al. Cellular responses regulated by rho-related small GTP-binding proteins. Philos Trans R Soc Lond B 1993;340:267-271.

31. Ridley AJ, Paterson HF, Johnston $\mathrm{CL}$, et al. The small GTP-binding protein rac regulates growth factor-induced membrane ruffling.[see comment]. Cell 1992;70:401-410.

32. Van Aelst L, D'Souza-Schorey C. Rho GTPases and signaling networks. Genes Dev 1997:11:2295-2322.

33. Nobes CD, Hall A. Rho, rac and cdc42 GTPases: regulators of actin structures, cell adhesion and motility. Biochem Soc Trans 1995;23:456-459.

34. Ridley AJ, Hall A. Distinct patterns of actin organization regulated by the small GTP-binding proteins Rac and Rho. Cold Spring Harb Symp Quant Biol 1992;57:661-671.

35. Waterman-Storer CM, Worthylake RA, Liu BP, et al. Microtubule growth activates Rac1 to promote lamellipodial protrusion in fibroblasts.[see comment]. Nat Cell Biol 1999;1:45-50.

36. Vaidya RJ, Ray RM, Johnson LR. MEK1 restores migration of polyaminedepleted cells by retention and activation of Rac1 in the cytoplasm. Am J Physiol Cell Physiol 2005;288:C350-C359.

37. Sander EE, van Delft S, ten Klooster JP, et al. Matrix-dependent Tiam1/ Rac signaling in epithelial cells promotes either cell-celladhesion or cell migration and is regulated by phosphatidylinositol 3-kinase. J Cell Biol 1998;143:1385-1398.

38. Kaplan J. Mechanisms of cellular iron acquisition: another iron in the fire. Cell 2002;111:603-606.

39. Neilands JB. Siderophores: structure and function of microbial iron transport compounds. J Biol Chem 1995;270:26723-26726.

40. van Renswoude J, Bridges KR, Harford JB, et al. Receptor-mediated endocytosis of transferrin and the uptake of Fe in $\mathrm{K} 562$ cells: identification of a nonlysosomal acidic compartment. Proc Natl Acad Sci USA 1982;79:6186-6190.

41. Yang J, Goetz D, Li JY, et al. An iron delivery pathway mediated by a lipocalin. Mol Cell 2002;10:1045-1056.

42. Goetz DH, Holmes MA, Borregaard N, et al. The neutrophil lipocalin NGAL is a bacteriostatic agent that interferes with siderophoremediated iron acquisition. Mol Cell 2002;10:1033-1043. 
43. Devireddy LR, Teodoro JG, Richard FA, et al. Induction of apoptosis by a secreted lipocalin that is transcriptionally regulated by IL-3 deprivation. Science 2001;293:829-834.

44. Kamezaki K, Shimoda K, Numata A, et al. The lipocalin 24p3, which is an essential molecule in IL-3 withdrawal-induced apoptosis, is not involved in the G-CSF withdrawal-induced apoptosis. Eur J Haematol 2003;71:412-417.

45. Klausen P, Niemann CU, Cowland JB, et al. On mouse and man: neutrophil gelatinase associated lipocalin is not involved in apoptosis or acute response. Eur J Haematol 2005;75:332-340.

46. Martin $M$, Simon-Assmann $P$, Kedinger $M$, et al. DCC regulates cell adhesion in human colon cancer derived HT-29 cells and associates with ezrin. Eur J Cell Biol 2006;85:769-83.

47. Braga VM, Del Maschio A, Machesky L. Regulation of cadherin function by Rho and Rac: modulation by junction maturation and cellular context. Mol Biol Cell 1999;10:9-22.

48. Kuroda S, Fukata M, Nakagawa $M$, et al. Role of IQGAP1, a target of the small GTPases Cdc42 and Rac1, in regulation of E-cadherin-mediated cell-celladhesion. Science 1998;281:832-835.
49. Ray ME, Mehra R, Sandler HM, et al. E-cadherin protein expression predicts prostate cancer salvage radiotherapy outcomes. J Urol 2006;176(Part 1):1409-1414; discussion 14.

50. Takaishi K, Sasaki T, Kotani H, et al. Regulation of cell-celladhesion by rac and rho small G proteins in MDCK cells. J Cell Biol 1997;139:1047-1059.

51. Hvidberg V, Jacobsen C, Strong RK, et al. The endocytic receptor megalin binds the iron transporting neutrophil-gelatinase-associated lipocalin with high affinity and mediates its cellular uptake. FEBS Lett 2005;579:773-777.

52. Bilello JP, Cable EE, Isom HC. Expression of E-cadherin and other paracellular junction genes is decreased in iron-loaded hepatocytes. Am J Pathol 2003;162:1323-1338.

53. Hanai J, Mammoto $T$, Seth $P$, et al. Lipocalin 2 diminishes invasiveness and metastasis of Ras-transformed cells. J Biol Chem 2005;280: 13641-13647.

54. Lee HJ, Lee EK, Lee KJ, et al. Ectopic expression of neutrophil gelatinase-associated lipocalin suppresses the invasion and liver metastasis of colon cancer cells. Int J Cancer 2006;118:2490-2497. 